\title{
Scaling relations for brittle fracture of entangled polystyrene melts and solutions in elongational flow
}

Wagner, Manfred H.; Narimissa, Esmaeil; Huang, Qian

Published in:

Journal of Rheology

Link to article, DOI:

$10.1122 / 8.0000184$

Publication date:

2021

Document Version

Peer reviewed version

Link back to DTU Orbit

Citation (APA):

Wagner, M. H., Narimissa, E., \& Huang, Q. (2021). Scaling relations for brittle fracture of entangled polystyrene melts and solutions in elongational flow. Journal of Rheology, 65(3), 311-324. https://doi.org/10.1122/8.0000184

\section{General rights}

Copyright and moral rights for the publications made accessible in the public portal are retained by the authors and/or other copyright owners and it is a condition of accessing publications that users recognise and abide by the legal requirements associated with these rights.

- Users may download and print one copy of any publication from the public portal for the purpose of private study or research.

- You may not further distribute the material or use it for any profit-making activity or commercial gain

- You may freely distribute the URL identifying the publication in the public portal 


\title{
Scaling Relations for Brittle Fracture of Entangled Polystyrene Melts and Solutions in Elongational Flow
}

\author{
Manfred H. Wagner ${ }^{1}$, Esmaeil Narimissa ${ }^{2,3}$, and Qian Huang ${ }^{4}$ \\ ${ }^{1}$ Polymer Engineering/Polymer Physics, Berlin Institute of Technology (TU Berlin), \\ Fasanenstrasse 90, 10623 Berlin, Germany \\ ${ }^{2}$ Dept. of Chemical Engineering, Technion-Israel Institute of Technology (IIT), Technion \\ City, Haifa 32 000, Israel \\ ${ }^{3}$ Dept. of Chemical Engineering, Guangdong Technion-Israel Institute of Technology \\ (GTIIT), Shantou 515063, China \\ ${ }^{4}$ Department of Chemical and Biochemical Engineering, Technical University of Denmark, \\ 2800 Kongens Lyngby, Denmark
}

\begin{abstract}
The criterion for brittle fracture of entangled polymer liquids [Journal of Rheology 62, 221 (2018)] is extended by including the effects of finite chain extensibility and polymer concentration. Crack initiation follows from rupture of primary $\mathrm{C}-\mathrm{C}$ bonds, when the strain energy of entanglement segments reaches the energy of the covalent bond. Thermal fluctuations will concentrate the strain energy on one C-C bond of entanglement segments, leading to bond scission and rupture of polymer chains followed by crack initiation and fast crack growth. In start-up flows, entanglement segments characterized by long relaxation times, i.e., predominantly those in the middle of the polymer chain will be the first to reach the critical strain energy and will fracture. Recent experimental data of Huang [Phys. Fluids 31, 083105 (2019)] of fracture of a monodisperse polystyrene melt and of several solutions of monodisperse polystyrenes dissolved in oligomeric styrene are in agreement with the scaling relations for critical Weissenberg number as well as Hencky strain and stress at fracture derived from this fracture criterion and the Extended Interchain Pressure model [Journal of Rheology 64, 95 (2020)].
\end{abstract}

Keywords: Polymer Melt; Polymer Solution; Fracture; Failure; Chain Scission; Elongation; EIP model; Interchain pressure; Finite Extensibility 


\section{INTRODUCTION}

Failure of polymeric liquids during elongational flow is not only of significant concern for the polymer industry when processes with free surfaces such as blow moulding, fibre spinning, film blowing, and paint spray are involved, but its understanding is also a scientific challenge. Moreover, defects such as melt fracture and sharkskin seen in extrusion processes are directly linked to this phenomenon. Consequently, failure has been the subject of many experimental, theoretical, and computational research endeavours for a long time, while progress in understanding of failure has been limited. A short account of experimental works and theoretical concepts is given in our 2018 publication [1]. Progress was hampered by the fact that failure includes the phenomena of ductile failure ("necking") and cohesive failure ("rupture" or "brittle fracture"), and until recently, the experimental separation of these two fundamentally different failure modes has been difficult or even impossible. This has changed with the advent of the filament stretching rheometer with locally controlled deformation and deformation rate developed by Hassager and coworkers, which is now commercially available (VADER 1000, Rheo Filament ApS [2]). By measuring the local diameter of the polymer sample during elongation, the true Hencky strain and strain rate can be determined and controlled, while by elongational rheometers prescribing the global deformation of the filament only nominal values of strain and strain rate can be obtained. Using a filament stretching rheometer, Huang et al. [3], and Huang and Hassager [4] showed that when true Hencky strain rate is controlled rather than nominal Hencky rate, the four failure zones of the so-called Malkin plot [5] (purely viscous zone, viscoelastic zone with failure by necking, rubbery zone, glassy zone) are reduced to just two possible states: liquid or solid, and a clear distinction exists between liquid behaviour (unlimited stead-state elongation) and solid behaviour (brittle fracture). For monodisperse polystyrene solutions and a polydisperse polystyrene melt they found that the value of the critical stress at fracture lies between the plateau $\left(G_{N}\right)$ modulus and the glassy $\left(G_{g}\right)$ modulus, and is at least 2 orders of magnitude higher than $G_{N}$. They reported that the critical strain remains approximately constant and the critical stress increases only slightly with increasing elongation rate. They also confirmed the high reproducibility of the critical stress/strain values, and demonstrated the simultaneous initiation and propagation of multiple cracks at different spatial positions along the stretched filament.

Considering the origin of crack initiation in polymeric systems, the idea of the largest preexisting microcrack (i.e. weakest-link), inherited from the Griffith theory [6], is not in 
agreement with the critical stress/strain data of Huang and Hassager [3, 4], which show a very high reproducibility. This leaves as possible origins of crack initiation either catastrophic chain disentanglement or rupture of primary $\mathrm{C}-\mathrm{C}$ bonds of the polymer chain. Main-chain scission in solid polymers, in polymer solutions and polymer melts is well documented. In amorphous and partially crystalline polymers below the glass transition temperature free radicals produced by chain scission can be identified by electron spin resonance (ESR) [49, 50]. The importance of polymer chain entanglements for brittle fracture of solid polymers was shown by Mikos and Peppas [51]. They found their calculated value of $1.0 \cdot 10^{17}$ polystyrene chains per square meter crossing the fracture surface to be in good agreement with experimental evidence of tensile strength, resulting from rupture of primary bonds and not from secondary (van der Waals) forces. Zhurkov and Korsukov [52] documented chain scission under tensile stress in polyethylene and polypropylene at temperatures up to $80^{\circ} \mathrm{C}$ by infrared spectroscopy, where free radicals can no longer be detected by conventional ESR due to fast recombination. Tabata et al. [53] showed direct evidence for main-chain scission of polymers in semidilute solution caused by high speed stirring using ESR combined with a spin trapping technique. Following earlier works (see e.g. Rehner [54] and references therein), Bestul $[55,56]$ investigated shear degradation in capillary flow by measuring intrinsic viscosities at successive stages of extrusion of concentrated solutions of polyisobutenes in cetane, and discussed how intra- and intermolecular configurational entropy and potential energy can be concentrated on one "bond-to-be-ruptured". Ballauf and Wolf [57] studied the degradation of solutions of 4.9-20 wt\% of polystyrene in trans-decalin by use of a shear cell at shear rates of $5.0 \cdot 10^{3}<\dot{\gamma}<10^{4} s^{-1}$. They showed that only a Gaussian breakage probability of $\mathrm{C}-\mathrm{C}$ bonds with the center of the probability distribution at the midpoint of the chain can reproduce the experimentally observed changes in the molar mass distribution. Mechanical degradation during multiple extrusion cycles in the melt state is well documented for polypropylene [58-61]: The molecular mass and polydispersity reduction as well as the lack of carbonyl groups and gel formation show that chain scission and not oxidation is the dominant degradation reaction. Degradation increases with increasing extrusion rate. Molar mass distribution curves suggest that chain scission occurs close to the middle part of the macromolecule, in agreement with the Bueche theory [62] for mechanical degradation. In controlled rheology experiments, Wang and co-workers [7] reported that beyond a critical strain rate, entangled melts and solutions undergo a yield-to-rupture transition. Birefringence measurements indicate that rupture occurs when the strands between entanglements undergo 
non-Gaussian stretching on their path to full extension, and the linear stress-optical rule starts to break down. They concluded that rupture occurs plausibly through chain scission in the limit of finite chain extensibility.

A novel and quantitative criterion of brittle fracture of entangled polymer liquids called the "entropic fracture hypothesis" was presented by the present authors [1]: Crack initiation follows from scission of primary C-C bonds, when the strain energy of an entanglement segment reaches the energy of the covalent bond. Thermal fluctuations lead to short-time concentration of the strain energy on one $\mathrm{C}-\mathrm{C}$ bond of the entanglement segment, and the chain ruptures. In start-up flows, entanglement segments characterized by long relaxation times, i.e., predominantly those in the middle of the polymer chain will be the first to reach the critical strain energy and will fracture. The entropic fracture hypothesis was shown to be in good qualitative agreement with experimental fracture data, but we already noticed that for quantitative agreement finite extensibility effects must be considered.

In a recent paper, Huang [8] investigated systematically the critical strain and stress at fracture for two groups of nearly monodisperse linear polystyrene liquids in controlled elongational flow. The samples in Group A have a similar number $Z$ of entanglements per chain but a different number $N_{e}$ of Kuhn segments or "monomers" per entanglement strand, while the samples in Group B have different $Z$ but similar $N_{e}$. Huang found no obvious dependence of the critical strain at fracture on $N_{e}$ and $Z$. By contrast, the critical stress at fracture is clearly independent of the number $Z$ of entanglements per chain, but influenced by the number $N_{e}$ of Kuhn monomers per entanglement strand.

In the present paper, we analyze the comprehensive data set of Huang [8] by use of the entropic fracture hypothesis, which we extend by taking finite extensibility and polymer fraction of the solutions into account. The paper is organized as follows: We first give a short account of the experimental data and the linear-viscoelastic characterization of the polymer systems considered. Then the Extended Interchain Pressure (EIP) model with finite chain extensibility is presented, followed by the fracture criterion and the resulting scaling relations. A new paradigm of finite extensibility is suggested, followed by comparison of fracture data and model predictions. 


\section{EXPERIMENTAL DATA AND LVE CHARACTERZATION}

\section{Materials}

The molecular characteristics of the polystyrene (PS) melt and solutions are summarised in Table 1 and are taken from Huang [8]. The samples are named in the form of PS-X/4k-Y, where $\mathrm{X}$ is the weight average molecular weight of the PS, 4k is the molecular weight of the oligomeric styrene solvent, and Y\% is the volume fraction of the PS in the solution. Group A contains three PS samples with different volume fractions (100\%, 33\%, and 18\%), namely, PS-285k, PS-864k/4k-33, and PS-1760k/4k-18. The samples have similar numbers $Z$ of entanglements, but different numbers $N_{e}$ of Kuhn monomers. Group B contains three PS solutions with similar volume fractions $(17 \%-18 \%)$ of polymer, namely, PS-1760k/4k-18, PS-864k/4k-17, and PS-576k/4k-17. These samples have similar values of $N_{e}$ but different $Z$.

\section{Mechanical Spectroscopy}

The linear viscoelastic properties of the PS samples were obtained from small amplitude oscillatory shear flow measurements using an ARES-G2 rheometer from TA Instruments. An $8 \mathrm{~mm}$ plate-plate geometry (with a sample thickness around $0.8 \mathrm{~mm}$ ) was used for lower frequencies and a $4 \mathrm{~mm}$ plate-plate geometry (with a sample thickness around $0.5 \mathrm{~mm}$ ) was used for higher frequencies in the transition to the glassy regime. The measurements were performed at temperatures $5 \mathrm{~K}$ (i.e. in the glassy regime) to $80 \mathrm{~K}$ higher than the corresponding glass transition temperatures of the samples. For each sample, the data were shifted to a single master curve at the reference temperature of $130^{\circ} \mathrm{C}$ using the time-temperature superposition procedure [8].

\section{Elongational Viscosity Measurements}

The stress-strain measurements in elongational flow were performed using a homemade filament stretching rheometer (FSR) [8]. An online control scheme was employed in the FSR to control the mid-filament diameter during stretching, so that the local strain rate was ensured to be constant. It should be noted that necking instabilities in Zones II and III in the Malkin failure master curve [5] can be controlled by the online control scheme in the FSR. Therefore, the four zones in the Malkin master curve are reduced to just two states: steady flow and elastic fracture [3, 4]. All the measurements were performed at $120-130^{\circ} \mathrm{C}$, and shifted to the reference temperature of $130^{\circ} \mathrm{C}$. 


\section{$\underline{\text { LVE Characterization }}$}

From linear-viscoelastic mastercurves of G' and G' (see Supplementary Material Fig. S1), continuous Baumgärtel-Schausberger-Winter (BSW) relaxation spectra [9] were determined and reported by Huang [8]. The relaxation modulus $G(t)$ is found from the spectrum $H(\tau)$ by

$$
G(t)=\int_{0}^{\infty} \frac{H(\tau)}{\tau} \exp (-t / \tau) d \tau
$$

$H(\tau)$ is composed of two part, the entanglement contribution $H_{e}(\tau)$ and the glassy contribution $H_{g}(\tau)$

$$
H(\tau)=H_{e}(\tau)+H_{g}(\tau)
$$

with

$$
H_{e}(\tau)=n_{e} G_{N}\left(\frac{\tau}{\tau_{t}}\right)^{n_{e}} h\left(1-\tau / \tau_{t}\right)
$$

and

$$
H_{g}(\tau)=n_{e} G_{N}\left(\frac{\tau}{\tau_{c}}\right)^{-n_{g}} h\left(1-\tau / \tau_{t}\right)
$$

$G_{N}$ is the plateau modulus, $\tau_{t}$ the longest or "terminal" relaxation time, $\tau_{c}$ the characteristic time constant of the glassy contribution (related to the entanglement segment equilibration time $\tau_{e}$ ), and $h(x)$ the Heaviside step function. The values of $n_{e}$ and $n_{g}$ are fixed to 0.23 and 0.70 , respectively. The physical meaning of the BSW parameters can be found elsewhere [10], and the BSW parameters of the polymer melts considered are given in Huang $[8]$.

The zero-shear viscosity of the samples is related to the BSW parameters by

$$
\eta_{0}=\int_{0}^{\infty} G(s) d s=n_{e} G_{N} \tau_{t}\left(\frac{1}{1+n_{e}}+\frac{1}{1-n_{g}}\left(\frac{\tau_{c}}{\tau_{t}}\right)^{n_{g}}\right) \cong n_{e} G_{N} \tau_{t}\left(\frac{1}{1+n_{e}}\right)
$$

As $\tau_{c}<<\tau_{t}$, the glassy contribution to $\eta_{0}$ can be neglected. From the plateau modulus $G_{N}$ of the samples the entanglement molar mass is calculated as [10]

$$
M_{e}=\frac{\rho R T}{G_{N}} \varphi
$$


$\rho$ is the density of polymer melt at the testing temperature (for PS taken as $1000 \mathrm{~kg} / \mathrm{m}^{3}$ ), $R$ is the gas constant, and $\varphi$ is the volume fraction of polymer in the solution with $\varphi=1$ specifying the melt. With $M$ being the molar mass of the polymer, the number of entanglements per chain, $Z$, is obtained,

$$
Z=\frac{M}{M_{e}}
$$

The relation between the entanglement molar mass of solution, $M_{e}$, and melt, $M_{e m}$, is given by

$$
M_{e}=M_{e m} \varphi^{-\alpha}
$$

The value of the dilution exponent $\alpha$ is model dependent $(1<\alpha<4 / 3)$, and a value of $\alpha=1$ was taken in [10]. The number of Kuhn segments or "monomers" between entanglements is $N_{e}=M_{e} / M_{0}$, where $M_{0}$ is the molar mass of the Kuhn monomer. For polystyrene melt, we take $M_{e m}=13300 \mathrm{~g} / \mathrm{mol}$ and $M_{0}=610 \mathrm{~g} / \mathrm{mol}[11]$.

While the BSW spectrum defined by Eqs. (1) to (4) allows for a reasonable quantification of the plateau modulus $G_{N}$, the BSW spectrum does not result in an ideal fit of the $G$ ' and $G$ ", mastercurves. We will therefore use parsimonious relaxation spectra here,

$$
G(t)=\sum_{i=1} g_{i} \exp \left(-t / \tau_{i}\right)
$$

for characterization of LVE in the experimentally accessible frequency window of all polymers considered. The partial moduli $g_{i}$ and relaxation times $\tau_{i}$ as determined by the IRIS software [12] result in excellent agreement with the linear-viscoelastic data of $G^{\prime}$ and G' (see Supplementary Material).

According to the DE model, the Rouse time $\tau_{R}$, the disengagement (or reptation) time $\tau_{d}$ and the zero-shear viscosity $\eta_{0}$ are given by [13],

$$
\tau_{R}=Z^{2} \tau_{e}
$$




$$
\begin{gathered}
\tau_{d}=3 Z \tau_{R} \\
\eta_{0}=\frac{\pi^{2}}{12} G_{N} \tau_{d}
\end{gathered}
$$

We identify here $\tau_{d}$ with the mean quadratic average of the relaxation times of the discrete relaxation spectrum and calculate $\eta_{\mathrm{o}}$ from the discrete relaxation spectrum,

$$
\begin{gathered}
\tau_{d}=\frac{\sum_{i} g_{i} \tau_{i}^{2}}{\sum_{i} g_{i} \tau_{i}} \\
\eta_{0}=\sum_{i} g_{i} \tau_{i}
\end{gathered}
$$

We note that Huang [8] assumed the equivalence of $\tau_{e}$ and $\tau_{c}$, i.e. $\tau_{e} \cong \tau_{c}$, and calculated the Rouse time in correspondence with Eq. (10) by the relation

$$
\tau_{R} \cong Z^{2} \tau_{c}
$$

We prefer to use Osaki's approach [14-17] for quantification of the Rouse time $\tau_{R}$, which extrapolates the Rouse time of unentangled polymer systems to the Rouse time of entangled polymer melts and solutions, and takes into account the power of 3.4 scaling of the zero-shear viscosity. This leads to the relation,

$$
\tau_{R}=\frac{12 M \eta_{0}}{\pi^{2} \rho R T \varphi}\left(\frac{M_{c m}}{M \varphi}\right)^{2.4}
$$

for the Rouse stretch relaxation time. $M_{c m}$ denotes the critical molar mass in the melt state. For monodisperse polystyrene we take the well-documented value of $M_{c m}=35 \mathrm{~kg} / \mathrm{mol}$ [18], and Eq. (16) has been used successfully for modeling of the transient and steady-state elongational and shear viscosities of PS melts and solutions [19, 20].

Tables 1 shows the molar mass $(M)$, polydispersity index $\left(M / M_{n}\right)$, glass transition temperature $\left(T_{g}\right)$, plateau modulus $\left(G_{N}\right)$, entanglement molar mass $\left(M_{e}\right)$, number of entanglements per chain $(Z)$, number of Kuhn monomers $\left(N_{e}\right)$ per entanglement, zero-shear viscosity $\left(\eta_{0}\right)$, 
terminal/reorientational/disengagement/reptation time $\left(\tau_{d}\right)$, Rouse stretch relaxation time $\left(\tau_{R}\right)$ of the monodisperse polymers samples considered in this study. The discrete relaxation spectra of all samples are reported in Table S1 (Supplementary Material).

Table 1. Molecular characterization $\left(M, M / M_{n}\right)$, glass transition temperature $\left(\mathrm{T}_{\mathrm{g}}\right)$, plateau modulus $\left(G_{N}\right)$, entanglement molar mass $\left(M_{e}\right)$, number of entanglements per chain $(Z)$, number of Kuhn monomers $\left(N_{e}\right)$ per entanglement, and material parameters $\eta_{0}, \tau_{d}$, and $\tau_{R}$ from linear-viscoelastic data using IRIS [12] spectra.

PS-285k PS-864k/4k-33 PS-1760k/4k-18 $\quad$ PS-864k/4k-17 $\quad$ PS-576k/4k-17

$\begin{array}{lccccc}\text { Group } & \text { A } & \text { A } & \text { A/B } & \text { B } & \text { B } \\ M[\mathrm{~kg} / \mathrm{mol}] & 285 & 864 & 1760 & 864 & 576 \\ \boldsymbol{M} / \boldsymbol{M}_{\boldsymbol{n}} & 1.09 & <1.15 & 1.13 & <1.15 & - \\ \boldsymbol{T}_{\boldsymbol{g}\left[{ }^{\circ} \mathrm{C}\right]} & 107.5 & 92.0 & 89.0 & 89.0 & 89.0 \\ \boldsymbol{G}_{N}[\mathrm{~Pa}] & 252040 & 25942 & 6850 & 6690 & 6890 \\ \boldsymbol{M}_{e}[\mathrm{~kg} / \mathrm{mol}] & 13.3 & 40.3 & 73.9 & 78.2 & 78.2 \\ Z_{[--]} & \mathbf{2 1 . 4} & \mathbf{2 1 . 0} & \mathbf{2 2 . 8} & 10.2 & 6.7 \\ \boldsymbol{N}_{e} & 21.8 & 66.1 & 121.1 & 128.2 & 128.2 \\ \boldsymbol{\eta}_{o}[\mathrm{MPa} \mathrm{s}] & 531 & 28.0 & 19.1 & 1.25 & 0.278 \\ \tau_{d}[\mathrm{~s}] & 8643 & 3695 & 11399 & 624 & 109 \\ \tau_{R}[\mathrm{~s}] & 360 & 173 & 252 & 73.8 & 29.7\end{array}$




\section{THE EXTENDED INTERCHAIN PRESSURE (EIP) MODEL}

The Molecular Stress Function (MSF) model is a generalized tube segment model with straindependent tube diameter [21-25]. The extra stress tensor $\sigma(t)$ of the MSF model with consideration of finite extensibility effects [26] is given by a history integral of the form

$$
\sigma(t)=\int_{-\infty}^{t} \frac{\partial G\left(t-t^{\prime}\right)}{\partial t^{\prime}} f \lambda \mathbf{S}_{D E}^{I A}\left(t, t^{\prime}\right) d t^{\prime}
$$

The strain measure $\mathbf{S}_{D E}^{I A}$ represents the contribution to the extra stress tensor originating from the affine rotation of the tube segments assuming "Independent Alignment (IA)" [27, 28], and is given by

$$
\mathbf{S}_{D E}^{I A}\left(t, t^{\prime}\right) \equiv 5\left\langle\frac{\mathbf{u}^{\prime} \mathbf{u}^{\prime}}{u^{\prime 2}}\right\rangle_{\mathrm{o}}=5 \mathbf{S}\left(t, t^{\prime}\right)
$$

with $\mathbf{S}\left(t, t^{\prime}\right)$ being the relative second order orientation tensor. $\mathbf{u}^{\prime} \mathbf{u}^{\prime}$ is the dyad of a deformed unit vector $\underline{\mathbf{u}}^{\prime}=\underline{\mathbf{u}}^{\prime}\left(t, t^{\prime}\right)$,

$$
\mathbf{u}_{-}^{\prime}=\mathbf{F}_{t}^{-1} \cdot \mathbf{u}
$$

$\mathbf{F}_{t}^{-1}=\mathbf{F}_{t}^{-1}\left(t, t^{\prime}\right)$ is the relative deformation gradient tensor, and $u^{\prime}$ is the length of $\mathbf{u}^{\prime}$. The orientation average is indicated by $<\ldots>0$,

$$
\langle\ldots\rangle_{\mathrm{o}} \equiv \frac{1}{4 \pi} \oiint[\ldots] \sin \boldsymbol{\theta}_{o} d \boldsymbol{\theta}_{o} d \boldsymbol{\varphi}_{o}
$$

i.e. an average over an isotropic distribution of unit vectors $\mathbf{u}$.

$\lambda=\lambda\left(t, t^{\prime}\right)$ represents the inverse of the relative tube diameter $a / a 0$, and at the same time the relative length of a deformed tube segment,

$$
\lambda\left(t, t^{\prime}\right)=\frac{a_{0}}{a\left(t, t^{\prime}\right)}=\frac{l\left(t, t^{\prime}\right)}{l_{0}}
$$

$t$ ' indicates the time when the tube segment was created by reptation processes with equilibrium tube diameter $a_{0}$ and equilibrium length $l_{0}$.

In the Gaussian limit, the molecular stress function $f=f\left(t, t^{\prime}\right)$ is equal to the tube stretch $\lambda$. However, this is valid only as long as $\lambda<0.5 \lambda_{\max }$ [29], where $\lambda_{\max } \cong \sqrt{N_{e}}$ represents the maximum stretch (i.e. a fully extended entanglement segment), and $N_{e}$ the number of Kuhn monomers in an entanglement segment. Outside the Gaussian regime, tension in the chain can be described by the inverse Langevin function, or, due to its mathematical complexity, approximations of it, like the so- called Warner [30] or Padé approximations [31]. Since the 
latter version seems to be more accurate, nonlinear elasticity caused by finite extensibility (FENE) is implemented in the EIP theory in the following way:

$$
f=c\left(\lambda, \lambda_{\max }\right) \lambda
$$

$c$ is a nonlinear spring coefficient, representing a relative Padé inverse Langevin function with [32]

$$
c=\frac{\left(3-\frac{\lambda^{2}}{\lambda_{\max }^{2}}\right) \cdot\left(1-\frac{1}{\lambda_{\max }^{2}}\right)}{\left(3-\frac{1}{\lambda_{\max }^{2}}\right) \cdot\left(1-\frac{\lambda^{2}}{\lambda_{\max }^{2}}\right)}
$$

We also tested second- and third-order functions presented recently by Howard [41] which are close to optimum in the sense of minimizing the magnitude of the maximum relative error in the approximation of the inverse Langevin function and found no significant difference to the use of Eq. (23).

We note that stretch $\lambda\left(t, t^{\prime}\right)$, tension $f\left(t, t^{\prime}\right)$, and orientation $\mathbf{S}\left(t, t^{\prime}\right)$ are relative quantities characterizing an entanglement segment created by reptation processes at time $t^{\prime}$ and still existing at time $t>t$ ' when the stress is measured. For simplicity, we drop the dependence on $t$ and $t^{\prime}$. While $\mathbf{S}_{D E}^{I A}$ is determined directly by the deformation history according to eq.(18), $\lambda$ is found as solution of an evolution equation taking into account affine tube segment deformation balanced by Rouse relaxation and the interchain pressure. In [1], we used an evolution equation for solutions, which did not take into account finite extensibility effects, and which characterized solutions by rescaling of the Rouse time due to the shift of the glass transition temperature. Here, we modify the stretch evolution equation of Narimissa et al. [19] by including the effect of finite extensibility into the interchain pressure term in the same way as explained in detail by Rolón-Garrído et al. [26],

$$
\frac{\partial \lambda}{\partial t}=\lambda(\kappa: \mathbf{S})-\frac{\lambda-1}{\tau_{R}}\left(1-\frac{2}{3} \varphi^{4}\right)-\frac{2 \varphi^{4}}{9 \tau_{R}} \lambda^{2}\left(\lambda f^{2}-1\right)
$$

with initial condition $\lambda\left(t=t^{\prime}, t^{\prime}\right)=1$. In eq.(24), the first term on the right hand sides describes an affine deformation, the second term Rouse relaxation, and the third term represents the interchain pressure contribution with Rouse time $\tau_{R}$ as defined by Eq. (16). Eq. (24) reduces to the evolution equation of the EIP model of Narimissa et al. [19] in the Gaussian limit, i.e when $c=1$ and $f=\lambda$. Eqs. (17) and (24) represent the EIP model with finite chain extensibility, and are solved numerically. 
From Eq. (24) follows in elongational flow at high Weissenberg numbers $W i=\dot{\varepsilon} \tau_{R}$ (with strain rate $\dot{\varepsilon}$ ) and large stretch, when full orientation (i.e. $\kappa: \mathbf{S}=\dot{\varepsilon}$ ) and equilibrium stretch (i.e. $\partial \lambda / \partial t=0$ ) are reached and the Rouse relaxation term can be neglected, that the product of molecular stress $f$ and stretch $\lambda$ is proportional to the square root of $W i$ and inverse proportional to the square of the polymer fraction $\varphi$,

$$
f \lambda=c \lambda^{2}=\frac{3}{2} \varphi^{-2} \sqrt{2 W i}
$$

and the tensile stress is expected to reach a value of,

$$
\sigma=5 G_{N} f \lambda=\frac{15}{2} G_{N} \varphi^{-2} \sqrt{2 W i}
$$

We note that in this asymptotic limit, the product $f \lambda$ does not depend on the finite extensibility function $c=c\left(\lambda, \lambda_{\max }\right)$, but is a sole function of polymer fraction $\varphi$ and $W i$.

From Eq. (26) and considering that $G_{N}=G_{N m} \varphi^{2}$, the universal relation for the high $W i$ tensile stress of melts and solutions of Narimissa et al. [19] is recovered,

$$
\sigma=\frac{15}{2} G_{N m} \sqrt{2 W i}
$$

with $G_{N m}$ being the plateau modulus of the melt.

\section{THE STRAIN ENERGY OF A POLYMER CHAIN IN A TUBE}

The entropic free energy $w\left(N_{e}\right)$ of a chain segment of a linear entangled chain with a large number of monomers $N_{e}$ (Kuhn segments or monomers with length b) in a tube with diameter $a$ is given by [33-35],

$$
w\left(N_{e}\right)=3 k T \frac{N_{e} b^{2}}{a^{2}}+c s t
$$

$k$ is Boltzmann's constant, and $T$ the absolute temperature. Taking the melt state (indicated by the index $m$ ) as reference state for the strain energy, $N_{e}=N_{e m}$ represents the number of monomers in an entanglement segment of length and diameter $a_{0 m}$ at equilibrium with $a_{o m}^{2}=N_{e m} b^{2}$. If the entropic free energy of an entanglement segment in the melt is scaled to the equilibrium thermal energy $3 k T$ by setting the constant $c s t$ to zero, $w\left(N_{e m}\right)$ represents the 
"interchain pressure energy" of the entanglement segment when squeezed by the neighbouring polymer chains in a tube with strain-dependent tube diameter $a \leq a_{\text {om }}$,

$$
w\left(N_{e m}\right)=3 k T \frac{a_{0 m}^{2}}{a^{2}}=3 k T \lambda_{m}^{2}
$$

In solutions, the number of monomers in the entanglement segment is increased by $N_{e}=N_{e m} \varphi^{-1}$ and the tube diameter at equilibrium is $a_{0}$ with $a_{o}^{2}=N_{e} b^{2}=a_{0 m}^{2} \varphi^{-1}$. Then the interchain pressure energy $w\left(N_{e}\right)$ is given by Eq. (28) as $w\left(N_{e}\right)=3 k T a_{0}^{2} / a^{2}=3 k T \lambda^{2}$, and from $w$ being proportional to the number of monomers, $w\left(N_{e}\right) \propto a_{0}^{2} \propto N_{e}$, it follows immediately that

$$
w\left(N_{e m}\right)=3 k T \frac{a_{0}^{2}}{a^{2}} \frac{N_{e m}}{N_{e}}=3 k T \lambda^{2} \varphi
$$

From the identity $\lambda^{2} \varphi=\frac{a_{0}^{2}}{a^{2}} \frac{a_{0 m}^{2}}{a_{0}^{2}}=\lambda_{m}^{2}$, we see that the strain energy $w\left(N_{e m}\right)$ is independent of the degree of dilution and depends only on the ratio of the equilibrium tube diameter $a_{0 m}$ in the melt state to the strain-dependent tube diameter $a$.

The strain energy increases with the square of chain stretch as long as Gaussian chain statistics applies. At larger deformations, finite extensibility effects cannot be neglected, and the tension increases over-proportionally with the stretch. In agreement with the stress tensor equation (17) we model this effect by use of the nonlinear spring constant $c$ of Eq. (23), resulting in a strain energy function of the form

$$
w\left(N_{e m}\right)=3 k T c \lambda^{2} \varphi=3 k T f \lambda \varphi
$$

\section{THE BOND ENERGY OF CARBON-CARBON BONDS AND THE FRACTURE CRITERION}

The bond energy of carbon-carbon bonds in hydrocarbons is reported as $348 \mathrm{~kJ} / \mathrm{mol}$ (see e.g. $[36,37])$, i.e. the bond energy of a single C-C bond with Avogadro's number $N_{A}=6.02 \cdot 10^{23}$ is: 


$$
U=\frac{348 \mathrm{~kJ}}{N_{A}}=5.78 \cdot 10^{-19} \mathrm{~J}
$$

On the other hand, the thermal energy $w_{e q}$ at a temperature $T$ of $393 K\left(120^{\circ} \mathrm{C}\right)$ is,

$$
w_{e q}=3 k T=1.62 \cdot 10^{-20} \mathrm{~J}
$$

with Boltzmann's constant $k=1.38 \cdot 10^{-23} J / K$. Thus, the bond energy $U$ is about 36 times larger than the thermal energy $w_{e q}$ at $120^{\circ} \mathrm{C}$, which is why the polymer chain will not rupture due to Brownian motion at equilibrium, while chain scission may be caused by ultraviolet (UV) radiation with more than $3.6 \mathrm{eV}$ [38].

The strain energy $w\left(N_{e m}\right)=3 k T f \lambda \varphi$ of a chain segment with $N_{e m}$ monomers is distributed along the segment. We make the hypothesis that when the strain energy of the segment reaches the critical energy

$$
w_{c}=3 k T f_{c} \lambda_{c} \varphi=U
$$

the total strain energy of the chain segment will be concentrated on one $\mathrm{C}-\mathrm{C}$ bond by thermal fluctuations, and this bond will then rupture. Ligoure and Mora [43] report thermal vibration frequencies in the range of $\omega_{t h}=10^{10}-10^{12} s^{-1}$, which include bond rotation and bond stretching. Using an Arrhenius ansatz, the lifetime of a C-C bond can be estimated as $\tau_{0}=\frac{1}{\omega_{t h}} \exp (U / k T)$. According to the Eyring kinetic theory of fracture, the lifetime also depends on the force $F$ carried by a link of length $\delta$, whose stored energy can supply a portion of the activation energy $[43,52]$. If we identify this link having a stored energy $F \delta$ with a chain segment carrying a strain energy $w$, the resulting bond lifetime reduces to $\tau=\frac{1}{\omega_{t h}} \exp \left(\frac{U-w}{k T}\right)=\tau_{0} \exp (-w / k T)$. Thus, when the strain energy $w$ reaches the bond energy $U$, the bond will rupture within a very short time. We note that while our fracture hypothesis is based on activation energy considerations, Wang et al. [44] presented a conceptual framework of force-coupled bond dissociation in the case of rubber failure recently. They consider the distortion of the covalent bond potential due to the effect of an external force, which reduces the energy barrier that the breaking bond needs to overcome by thermal fluctuations. 
We recall that stretch and tension are relative quantities, and therefore the strain energy $w=w\left(t, t^{\prime}\right)$ is also a relative quantity. In start-up flows segments with long relaxation times, i.e. those preferably in the middle of the chain, will be the first to reach the critical energy $w_{c}$ and will fracture. Chain segments closer to the ends of the chain, which due to reptation processes have shorter relaxation times and see less stretch and tension within the time interval of $t^{\prime}$ (creation) and $t$ (observation), will not reach $w_{c}$ and are less likely to fracture. This is in agreement with experimental evidence [57-61] presented in the Introduction. When a bond ruptures, the newly created ends of the chain segment will recoil within a segmental Rouse time, thereby dissipating the strain energy, which may then be transmitted to primary $\mathrm{C}-\mathrm{C}$ bonds of adjacent chains leading to further chain scissions. We assume that as soon as the strain energy $w=w\left(t, t^{\prime}=0\right)$ accumulated between the start-up of deformation at time $t^{\prime}=0$ and time $t=t_{c}$ reaches the critical energy $w_{c}=w\left(t=t_{c}, t^{\prime}=0\right)$, a sufficient concentration of locally ruptured chains is reached, i.e. the damage probability as e.g. defined by Verneray et al. [45] for rubber networks becomes equal to one and crack initiation will occur. Crack initiation is followed by crack growth, which leads within a very short time (about 200ms according to Huang et al. [3]) to brittle fracture of the polymer liquid. At time $t=t_{c}$, the critical Hencky strain at fracture, $\varepsilon_{c}=\dot{\varepsilon} t_{c}$, is reached and the critical tensile stress at fracture, $\sigma_{c}=\sigma\left(t_{c}\right)$, is given by the stress equation (17). Crack initiation can take place at several locations simultaneously along the polymer sample under tension, and high-speed images revealed indeed that multiple cracks propagate $[3,4]$, one of which leading to rupture of the sample, while the others close up elastically. Crack profiles and energy dissipation during crack propagation were investigated in detail by Huang et al. [3] and are not considered here.

From the fracture hypothesis defined by Eq. (34), the maximum achievable product of critical molecular stress $f_{c}$ and critical stretch $\lambda_{c}$ is obtained,

$$
f_{c} \lambda_{c} \varphi=c \lambda_{c}^{2} \varphi=\frac{U}{3 k T} \cong 36
$$

Eqs. (29) and (35) define a critical tube diameter $a_{c}$ in terms of the equilibrium tube diameter $a_{0 m}$ of the melt,

$$
a_{c} \cong \frac{1}{6} \sqrt{c} a_{0 m}
$$


at which the critical interchain pressure energy is reached and rupture of primary C-C bonds and thus brittle fracture is expected to occur. We called this fracture mode "entropic fracture" [1], as explained above it is caused by thermal fluctuations, and entanglement segments are not fully stretched when fracture occurs. This is in contrast to the "enthalpic" fracture hypothesis of Lake and Thomas [36] as modified by Mazich and Samus [39]. These authors assumed that all bonds are fully stretched at fracture and when a chain with $N \mathrm{C}-\mathrm{C}$ bonds between two entanglement points ruptures, the strain energy $w_{c}=N U$ corresponding to the bond energy of all $N$ C-C bonds in the entangled chain segment is dissipated. Instead of using this energy argument, one might also consider a corresponding force argument: Assuming a generally accepted bond fracture strength of $4 n N$, the critical stress needed to rupture the chains (again assuming fully stretched and oriented chain segments) would be more than an order of magnitude larger than the stress at rupture observed experimentally as shown in [1, 42].

Combining the fracture criterion of Eq. (35) with the asymptotic tensile stress at high Wi and high stretch according to Eq. (26), we expect at sufficiently fast and large elongations a critical tensile stress

$$
\sigma_{c}=5 G_{N} f_{c} \lambda_{c}=\frac{15}{2} G_{N} \varphi^{-2} \sqrt{2 W i_{c}} \cong 5 G_{N} \frac{U}{3 k T} \varphi^{-1}
$$

From the first and last term of this identity, we expect the following scaling relation for the normalized critical stress $\sigma_{c} \varphi / G_{N}$ at fracture,

$$
\frac{\sigma_{c} \varphi}{G_{N}} \cong 5 \frac{U}{3 k T} \cong 180
$$

The reduced critical Weissenberg number $W i_{c} / \varphi^{2}$ for the onset of fracture,

$$
\frac{W i_{c}}{\varphi^{2}} \cong \frac{2}{9}\left(\frac{U}{3 k T}\right)^{2} \cong 288
$$

is obtained from the two last terms of Eq. (37). Wi is the minimum Weissenberg number for fracture to occur, i.e. fracture is expected only for $W i \geq W i_{c}$. The characteristic numbers " 180 " and " 288 " in the scaling relations result from the combination of the fracture criterion with the EIP model. 


\section{A NEW PARADIGM OF FINITE EXTENSIBILITY}

Finite extensibility is defined as

$$
\lambda_{\max }^{2}=N_{e}=N_{e m} \varphi^{-1}
$$

and is considered to be a constant material property. However, the fracture data of Huang [8] reveal (as demonstrated below in Figs. 2a,b and 4) that finite extensibility is actually a dynamic property: At fast deformations with large $W i$, there is not sufficient time to reach equilibrium within the entanglement segment. The relevant $W i$ is therefore not $W i=W i_{R}=\dot{\varepsilon} \tau_{R}=\dot{\varepsilon} \tau_{e} Z^{2}$, which characterizes the full chain, but rather

$$
W i_{e}=\dot{\varepsilon} \tau_{e}=W i / Z^{2}
$$

$\tau_{e}$ is the equilibration time of an entanglement segment and increases with dilution in OS at iso- $\mathrm{T}_{\mathrm{g}}$ temperature according to $\tau_{e}=\tau_{e m} \varphi^{-2}$ [40]. Thus, while for $W i_{e}<1$ equilibrium in the entanglement segment of the solution is reached and $\lambda_{\max }^{2}=N_{e}=N_{e m} \varphi^{-1}$, the time scale at $W i_{e} \geq 1 / \varphi^{2}$ corresponding to $W i_{e m}=\dot{\varepsilon} \tau_{e m} \geq 1$ is set by $\tau_{e m}$, and $\lambda_{\max }^{2}$ reduces to $\lambda_{\max }^{2}=N_{e m}$. To account for the reduction of $\lambda_{\max }^{2}$ with increasing $W i_{e}$, we use the empirical relation

$$
\lambda_{\max }^{2}=N_{e m} \frac{1 / \varphi+a W i_{e}}{1+a W i_{e}}=N_{e m} \frac{Z^{2} / \varphi+a W i}{Z^{2}+a W i}
$$

$a$ is a numerical parameter, the only free parameter of the model. 


\section{COMPARISON OF THE FRACTURE HYPOTHESIS TO EXPERIEMTAL EVIDENCE}

In the following we compare predictions of our entropic fracture model for the origin of brittle fracture of entangled polymer liquids to the experimental data of Huang [8]. We consider first melt PS-285k and solutions PS-864k/4k-33 and PS-1760k/4k-18, which feature a similar number of entanglements $Z$, but widely differing numbers of Kuhn monomers $N_{e}$ per entanglement (Group A). This is followed by discussion of Group B comprising the three solutions PS-1760k/4k-18, PS-864k/4k-17, and PS-576k/4k-17, which have different numbers of entanglements, but similar numbers of Kuhn monomers.

\subsection{Polymer systems of Group A (PS-285k, PS-864k/4k-33 and PS-1760k/4k-18)}

Fig.1 shows the comparison between experimental data and model predictions of Hencky strain at break $\varepsilon_{c}$, reduced tensile stress $\sigma / G_{N}$ as well chain stretch $\lambda$, molecular tension $f$ and strain energy $w$ as a function of Weissenberg number $W i=\dot{\varepsilon} \tau_{R}$ for melt PS-285k. Fractured samples are indicated by full symbols and full lines, steady-state stress by open symbols and dotted lines for experimental data and model prediction, respectively. At higher $W i$ in the range of $60-300$, it is not clear whether PS-285k failed due to elastic fracture or due to failure of the online control scheme leading to fast necking [8]. Such points are presented as asterisks. For PS-285k, the number of entanglements is $Z=21.4$, and the number of Kuhn monomers $N_{e m}$ per entanglement is 21.8 and therefore $\lambda_{\max }^{2}=N_{e m}=21.8$. As demonstrated in Fig.1a, using this value of $\lambda_{\max }^{2}$ in equation (23) for the nonlinearity function $c=c\left(\lambda^{2}, \lambda_{\max }^{2}\right)$ leads to excellent agreement of data and model for the Hencky strain at break $\varepsilon_{c}$. The prediction of the onset of the reduced critical tensile stress at fracture $\sigma_{c} / G_{N}$ (Fig.1b) is in quantitative agreement with the data, while at higher Wi the predicted fracture stress increases (Fig.1b) when approaching the regime of the glassy modes with storage and loss modulus larger than $G_{N}$. As seen in Fig.1c, the entanglement stretch at fracture is limited to $\lambda=3.9$, which means that entanglements which fracture are not fully stretched, but reach $83 \%$ of maximal stretch $\lambda_{\max }=\sqrt{N_{e m}}=\sqrt{21.8}=4.7$. This is in agreement with earlier findings of Wang et al. [7] that chain scission occurs on the way to full chain extension. The tension in the entanglements reaches a maximum value of $f=9.3$, and the fracture criterion 
of maximum reduced free energy $w_{s}=w / 3 k T=f_{c} \lambda_{c} \cong 36$ is confirmed by comparison with the experimental data in Figs.1a and b.

Also shown in Fig. 1 are predictions for the Gaussian limit, i.e. for $\lambda_{\max }^{2}=N_{e m} \rightarrow \infty$, which we had considered earlier [1]. The Hencky strain at break $\varepsilon_{c}$ turns out to be larger (dashdotted line in Fig. 1a) and is clearly not in quantitative agreement with the experimental data. However, because the product $f \lambda$, the tensile stress $\sigma$, and the strain energy $w$ do not depend on the finite extensibility function $c=c\left(\lambda, \lambda_{\max }\right)$ at larger deformations (see Eqs. (25) and (26)), there is no significant change in the prediction of the reduced critical tensile stress at fracture $\sigma_{c} / G_{N}$. In the Gaussian limit, $\lambda=f=\lambda_{G}$ reaches a limiting value of $\lambda_{G}=\lambda_{c}=\sqrt{\frac{U}{3 k T}}=6$ according to Eq. (35) with $c=1$, which is larger than the maximum stretch $\lambda_{\max }=4.7$ of the entanglement segment (Fig. 1c). This shows the importance of taking into account finite extensibility in the evolution equation (24) of the stretch.

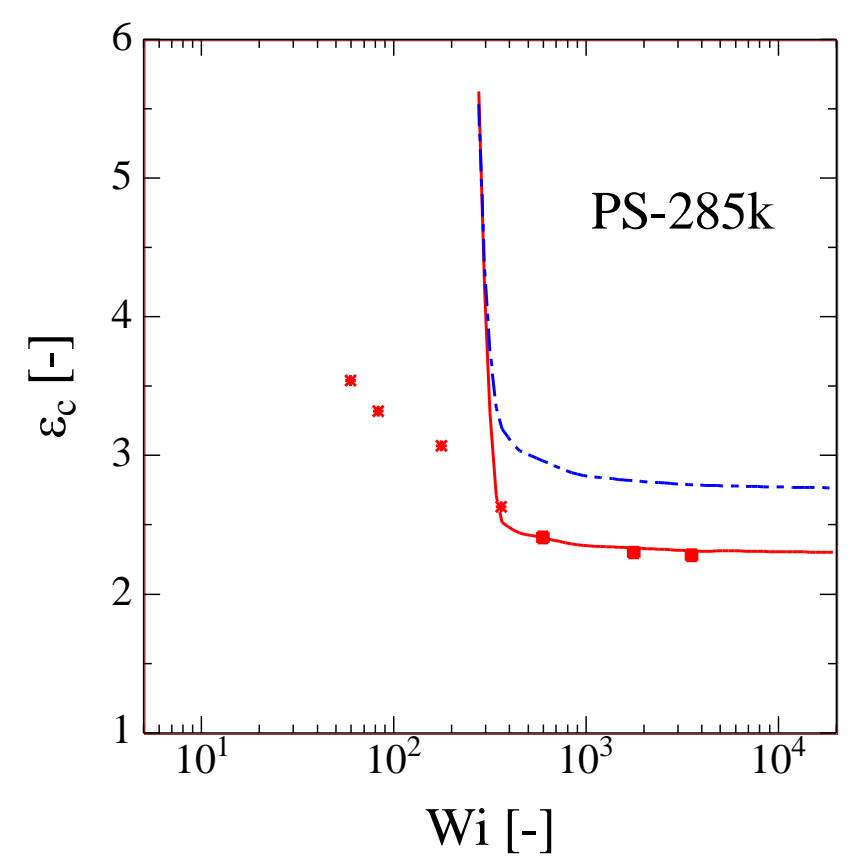

(a) 


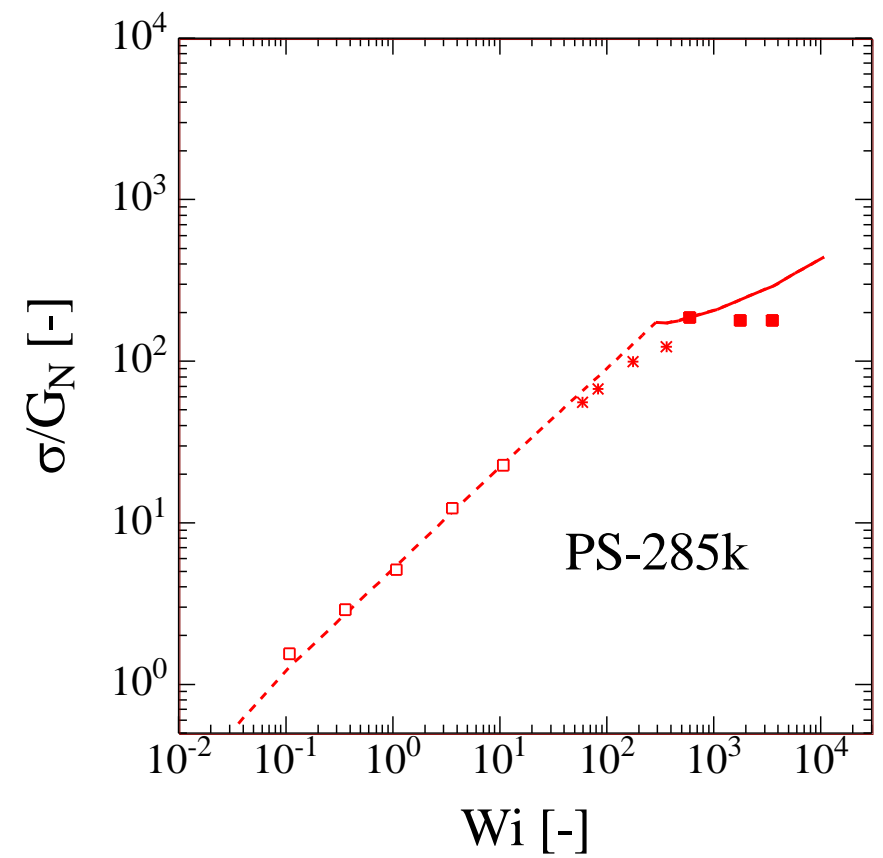

(b)

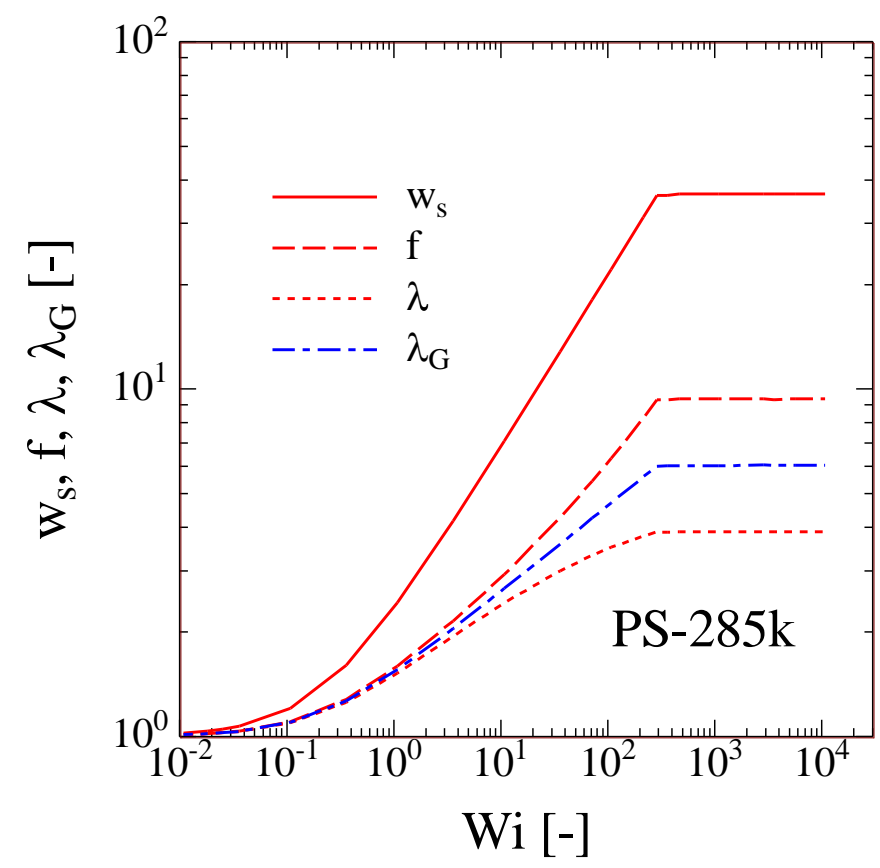

(c)

Fig.1: Comparison of data (symbols) and predictions (lines) as a function of Weissenberg number $W i=\dot{\varepsilon} \tau_{R}$ for melt PS-285k. Samples which failed by fast necking are presented as asterisks.

(a) Critical Hencky strain at break $\varepsilon_{c}$. Solid line is prediction for $\lambda_{\max }^{2}=N_{e m}=21.8$, dashdotted line is prediction for the Gaussian limit $\left(\lambda_{\max }^{2}=N_{e m} \rightarrow \infty\right)$.

(b) Reduced tensile stress $\sigma / G_{N}$. Fractured samples are indicated by full symbols and full lines, steady-state stress by open symbols and dotted lines.

(c) Normalized free energy $w_{s}=w / 3 k T$, tension $f$, stretch $\lambda$ of entanglement segment.

Dash-dotted line is prediction of stretch $\lambda_{G}$ for the Gaussian limit $\left(\lambda_{\max }^{2}=N_{e m} \rightarrow \infty\right)$. 
The solutions PS-864k/4k-33 and PS-1760k/4k-18 have similar numbers of entanglements as melt PS-285k with $Z=21$ (PS-864k/4k-33) and $Z=22.8$ (PS-1760k/4k-18), but the number of Kuhn segments per entanglement is much larger with $N_{e}=N_{e m} \varphi^{-1}=66.1$ for PS-864k/4k-33 and $N_{e}=121.1$ for PS-1760k/4k-18. As seen in Figs.2 a and b, using these values of $\lambda_{\max }^{2}=N_{e}$ in equation (23) for the nonlinearity function $c=c\left(\lambda^{2}, \lambda_{\max }^{2}\right)$ leads to the correct prediction of the onset of fracture at a critical Weissenberg number of $W i_{c} \approx 30$ and $W i_{c} \approx 12$, respectively, but at higher $W i$, the predictions of the Hencky strain at break $\varepsilon_{c}$ are constant and significantly higher than the values of $\varepsilon_{c}$ observed experimentally, which decrease with increasing Wi. Replacing $\lambda_{\max }^{2}$ in Eq. (23) by $\lambda_{\max }^{2}=N_{e m}=21.8$ does not affect the onset of fracture, but reduces the Hencky strain at break $\varepsilon_{c}$ to a level which is reached experimentally at high Wi. It is evident that $\lambda_{\max }^{2}$ decreases with increasing $W i$, and using the relation (42) with parameter $a=20$ for PS-864k/4k-33 and $a=2$ for PS-1760k/4k-18 leads to nearly quantitative agreement of data and prediction. As seen in Figs.2 $\mathrm{c}$ and d, the chain stretch at fracture reaches a maximum of $\lambda=5.6$ for PS-864k/4k-33, which corresponds to $69 \%$ of maximal stretch $\lambda_{\max }=8.1$. For PS- $1760 \mathrm{k} / 4 \mathrm{k}-18$, the maximal value is $\lambda=9.0$, which corresponds to $82 \%$ of maximal stretch $\lambda_{\max }=11.0$. Due to the decreasing $\lambda_{\max }^{2}$ with increasing $W i$ according to relation (42), the critical stretch $\lambda_{c}$ at break then decreases, while the molecular tension $f_{c}$ continues to increase. The net effect is a constant $w_{s}=f_{c} \lambda_{c} \varphi \cong 36$. In the Gaussian limit with $\lambda_{\max }^{2}=N_{e} \rightarrow \infty$ and $c=c\left(\lambda, \lambda_{\max }\right)=1, \lambda_{G}$ reaches a limiting value of $\lambda_{G}=\lambda_{c}=\sqrt{\frac{U / \varphi}{3 k T}}=6 \varphi^{-1 / 2}$ according to Eq. (35), corresponding to $\lambda_{c}=10.4$ and $\lambda_{c}=14.1$ for PS-864k/4k-33 and PS-1760k/4k-18, respectively, which is again larger than the maximum stretches (Fig.2 $\mathrm{c}$ and d). 


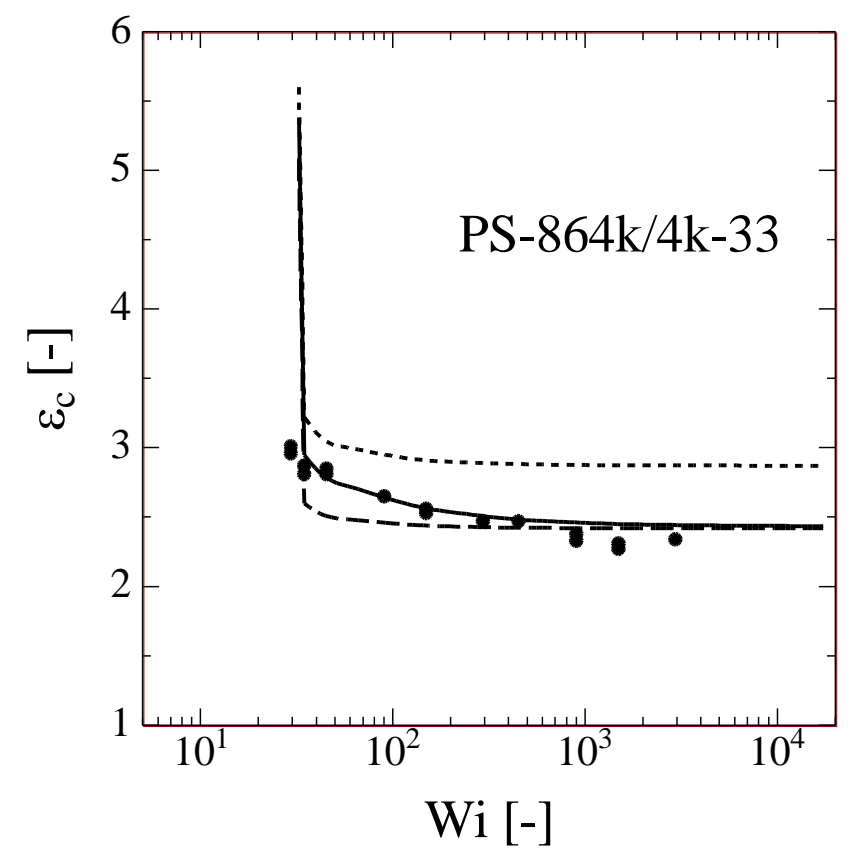

(a)

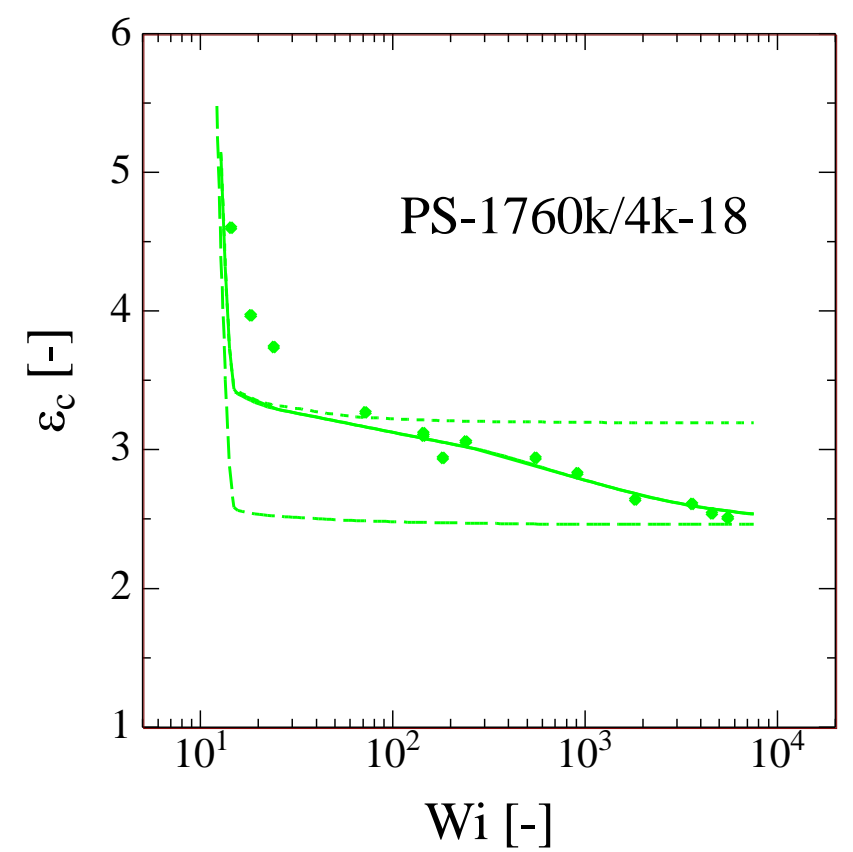

(b) 


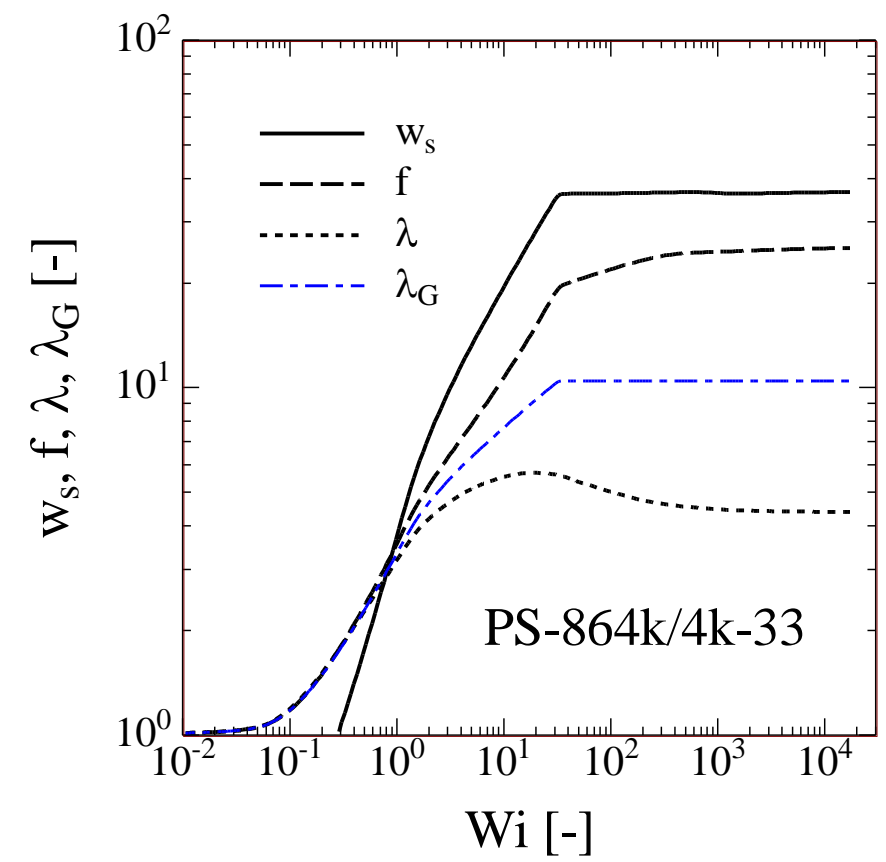

(c)

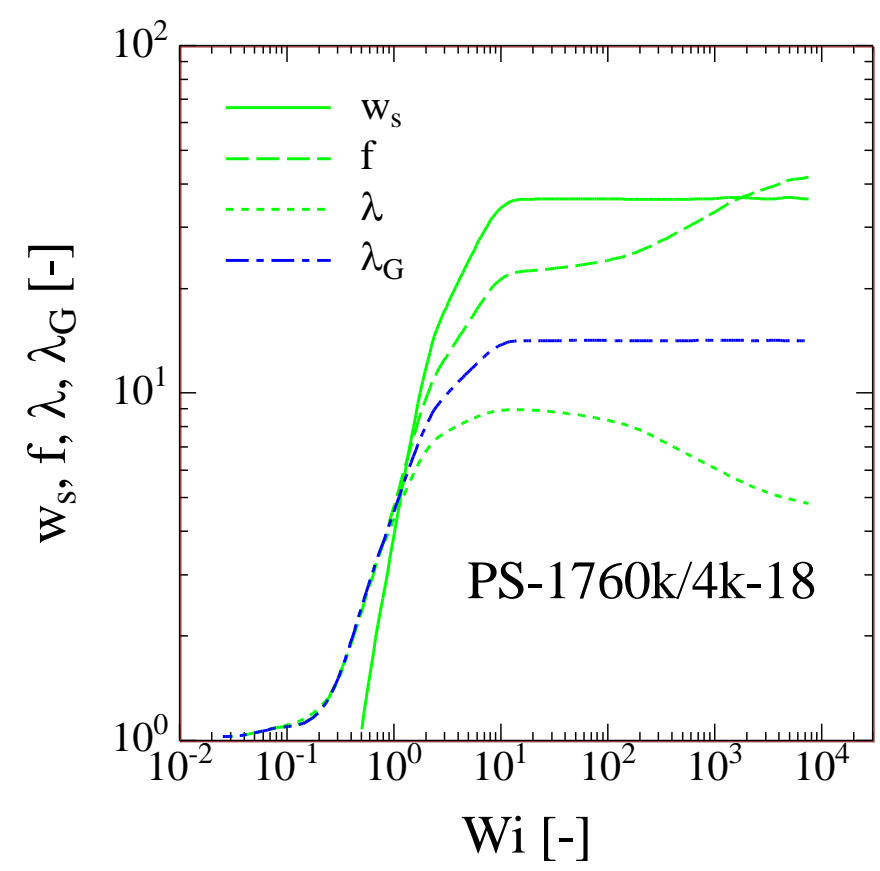

(d)

Figs.2 (a) and (b): Comparison of data (symbols) and predictions (lines) of critical Hencky strain at break $\varepsilon_{c}$ as a function of Weissenberg number $W i=\dot{\varepsilon} \tau_{R}$ for solutions PS-864k/4k-33 and PS-1760k/4k-18. Short and long dotted line predictions for $\lambda_{\max }^{2}=N_{e} \varphi^{-1}$ and $\lambda_{\max }^{2}=N_{e m}=21.8$, respectively. Full line prediction by Eq. (42) with parameter $a=20$ for PS$864 \mathrm{k} / 4 \mathrm{k}-33$ and $a=2$ for PS-1760k/4k-18.

(c) and (d): Normalized free energy $w_{s}=w / 3 k T$, tension $f$, stretch $\lambda$ of entanglement segment of solutions PS-864k/4k-33 and PS-1760k/4k-18. Dash-dotted line is prediction of stretch $\lambda_{G}$ for the Gaussian limit $\left(\lambda_{\max }^{2}=N_{e m} \rightarrow \infty\right)$. 
Fig.3 compares experimental data and model predictions of Hencky strain at break $\varepsilon_{c}$ and reduced tensile stress $\sigma / G_{N}$ for melt PS-285k and solutions PS-864k/4k-33 and PS-1760k/4k18. Fractured samples are indicated by full symbols, steady-state stress by open symbols. Fig.3a demonstrates that with increasing $N_{e}$ and decreasing polymer fraction $\varphi$ the critical Weissenberg number $W i_{c}$ at break decreases, while the critical stress at fracture increases (Fig.3b).

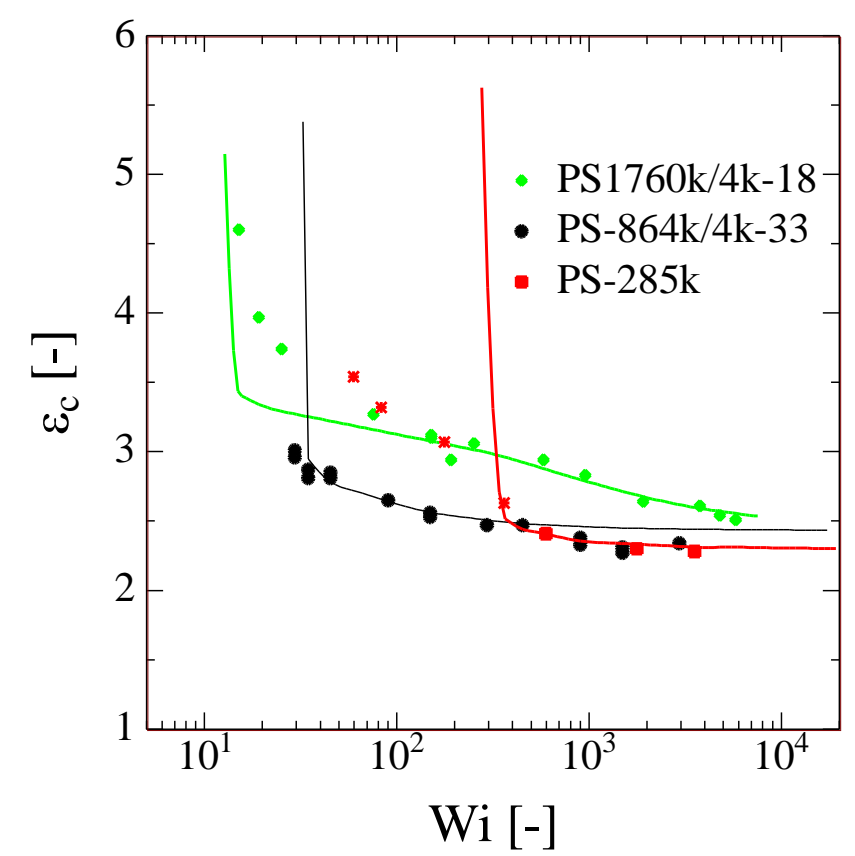

(a) 


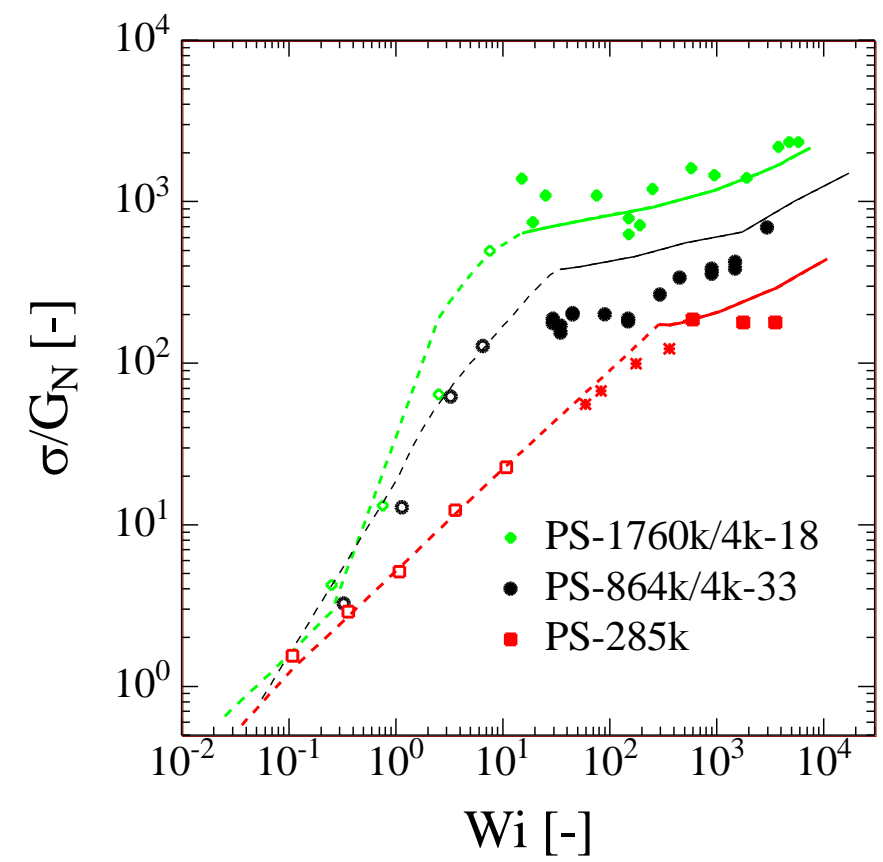

(b)

Fig.3: Comparison of data (symbols) and predictions (lines) as a function of Weissenberg number $W i=\dot{\varepsilon} \tau_{R}$ for melt PS-285k and solutions PS-864k/4k-33 and PS-1760k/4k-18. Samples which failed by fast necking are presented as asterisks.

(a) Critical Hencky strain at break $\varepsilon_{c}$,

(b) Reduced tensile stress $\sigma / G_{N}$. Fractured samples are indicated by full symbols and full lines, steady-state stress by open symbols and dotted lines.

\subsection{Solutions PS-864k/4k-17, PS-545k/4k-17, and PS-1760k/4k-18 (Group B)}

The three solutions of Group B have different numbers $Z$ of entanglements (Table I), but similar numbers of Kuhn monomers $N_{e}=N_{e m} \varphi^{-1}$ and therefore similar maximal stretches $\lambda_{\max }$ with $\lambda_{\max }^{2}=N_{e}=128.2$ for PS-864k/4k-17 and PS-545k/4k-17, and $\lambda_{\max }^{2}=N_{e}=121.1$ for PS-1670k/4k-18. As in the case of solution PS-1760k/4k-18 and shown in Fig.4, using $\lambda_{\max }^{2}=N_{e}=128.2$ in the equation (23) for the nonlinerarity function $c=c\left(\lambda^{2}, \lambda_{\max }^{2}\right)$ leads again to the correct predictions of the onset of fracture at a critical Weissenberg number of approximately $W i_{c} \approx 10$ for PS-864k/4k-17 and $W i_{c} \approx 12$ for PS-545k/4k-17, in spite of the different $Z$ of these solutions. Replacing $\lambda_{\max }^{2}$ in Eq. (23) by $\lambda_{\max }^{2}=N_{e m}=21.8$ does not affect the onset of fracture, but reduces the Hencky strain at break $\varepsilon_{c}$ to a level which is seen experimentally at high Wi. As shown in Fig.4, using relation (42) with the same parameter $a=2$ for all three solutions of Group B leads to reasonable agreement of data and prediction. 


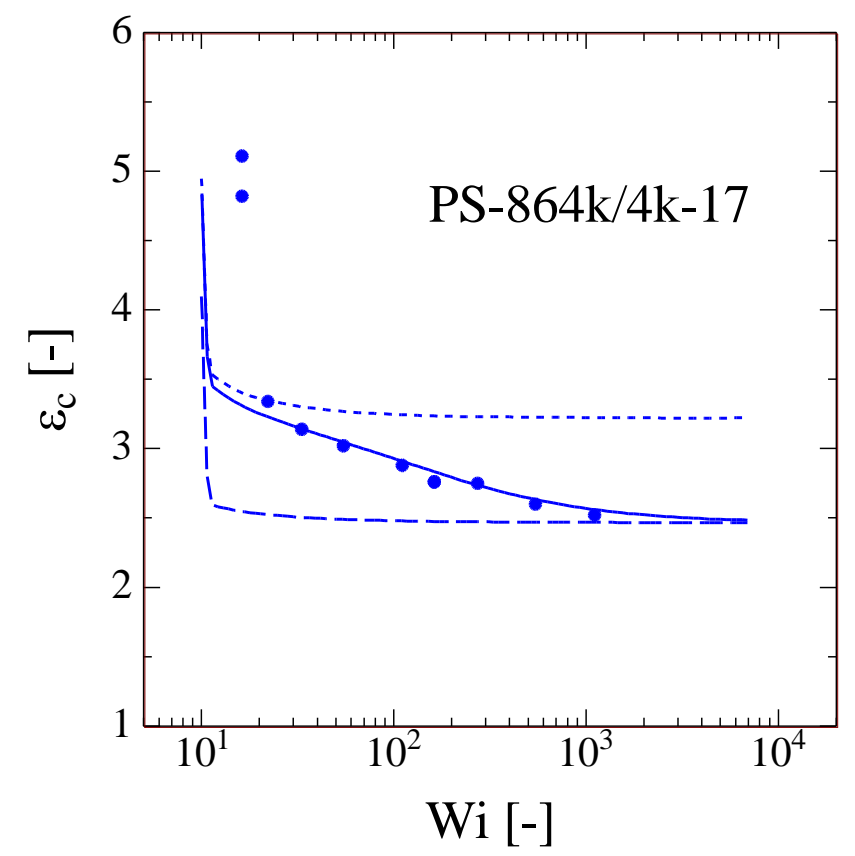

(a)

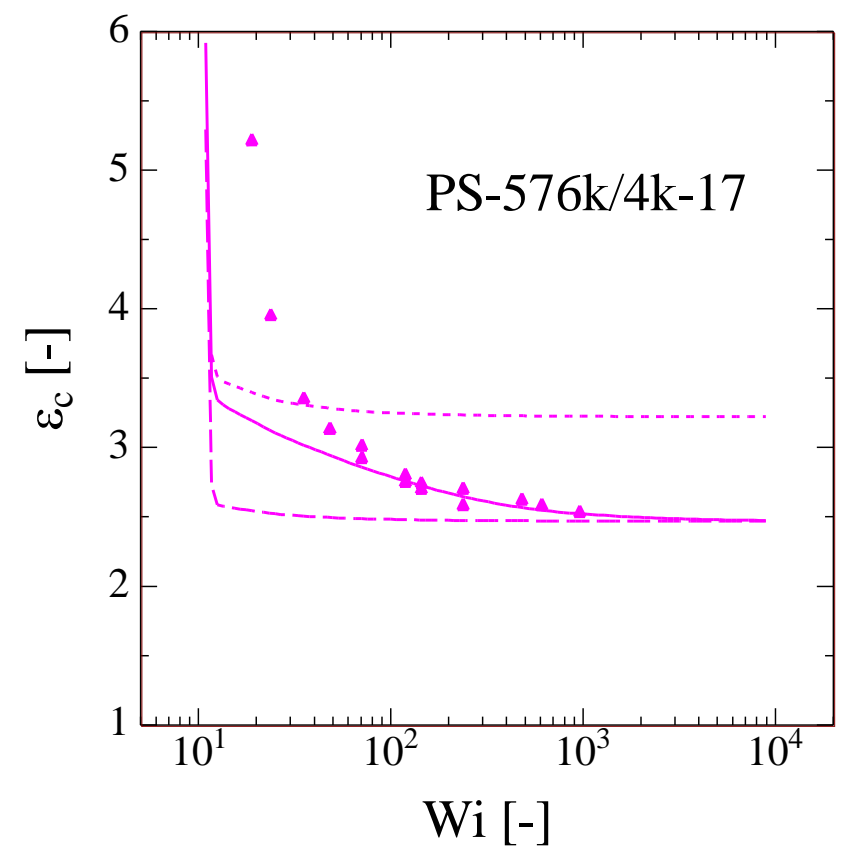

(b)

Fig.4: Comparison of data (symbols) and predictions (lines) of critical Hencky strain at break $\varepsilon_{c}$ as a function of Weissenberg number $W i=\dot{\varepsilon} \tau_{R}$ for solutions (a) PS-864k/4k-17 and (b) PS-576k/4k-17. Short and long dotted line predictions for $\lambda_{\max }^{2}=N_{e} \varphi^{-1}$ and $\lambda_{\max }^{2}=N_{e m}=21.8$, respectively. Full line prediction by Eq. (42) with parameter $a=2$. 
A comparison of the critical strain at break and the reduced tensile stress $\sigma / G_{N}$ is presented in Fig.5 for the three solutions of Group B with similar $N_{e}$ but different $Z$. As seen in Fig.5a, the critical Weissenberg number for the onset of fracture is approximately the same for all three solutions, largely independent of $Z$. The predicted reduced critical tensile stress at fracture $\sigma_{c} / G_{N}$ (full lines in Fig.5b) increases with increasing $W i$ in quantitative agreement with the experimental data (full symbols) and shows an enhanced increase with decreasing $Z$, which is related to the smaller width of the rubber-like plateau with decreasing $Z$.

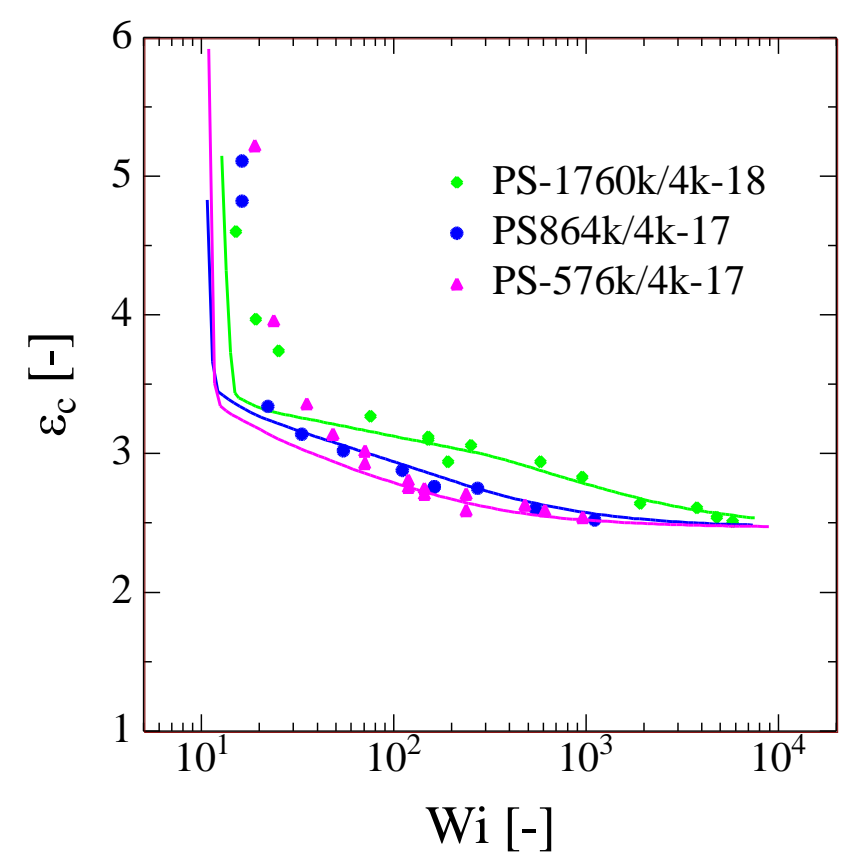

(a) 


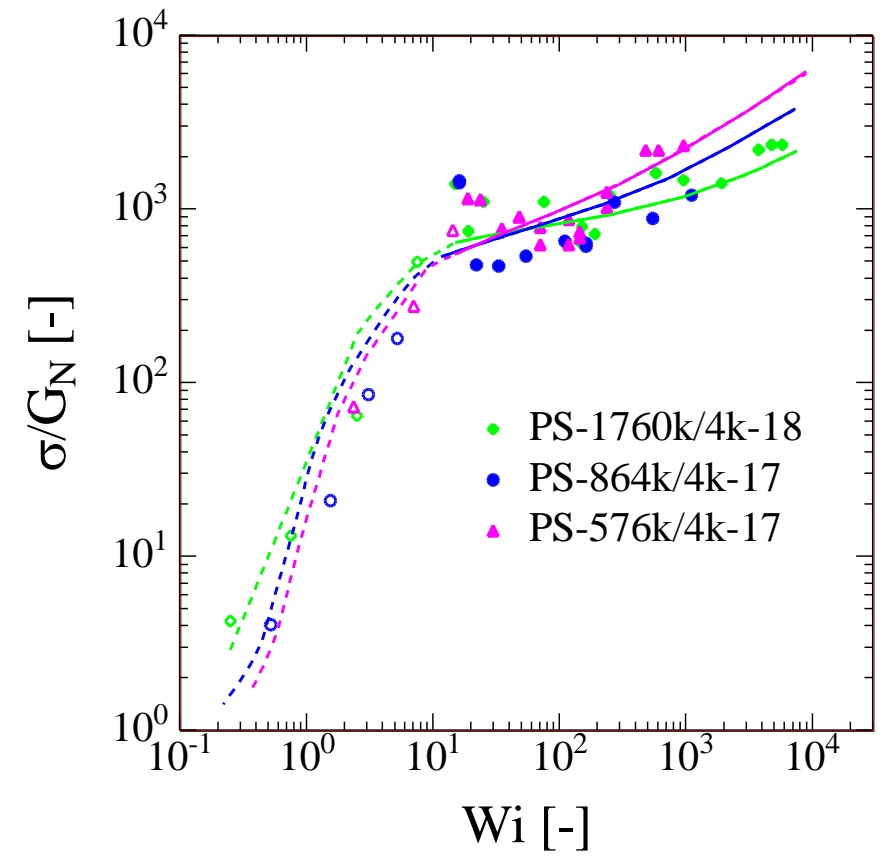

(b)

Fig.5: Comparison of data (symbols) and predictions (lines) as a function of Weissenberg number $W i=\dot{\varepsilon} \tau_{R}$ for solutions PS-1760k/4k-18, PS-864k/4k-17, and PS-576k/4k-17;

(a) Critical Hencky strain at break $\varepsilon_{c}$,

(b) Reduced tensile stress $\sigma / G_{N}$. Fractured samples are indicated by full symbols and full lines, steady-state stress by open symbols and dotted lines. 


\section{UNIVERSAL SCALING RELATIONS FOR FRACTURE OF PS MELTS AND SOLUTIONS IN ELONGATIONAL FLOW}

Fig. 6 presents the critical strain at fracture as a function of the reduced Weissenberg number $W i / \varphi^{2}$. The reduced critical Weissenberg number $W i_{c} / \varphi^{2}$ for the onset of fracture given by Eq. (39), $W i_{c} / \varphi^{2}=288$, is indicated in the figure by the vertical dotted line. Fracture occurs only at or to the right of this universal line for the melt and all solutions investigated.

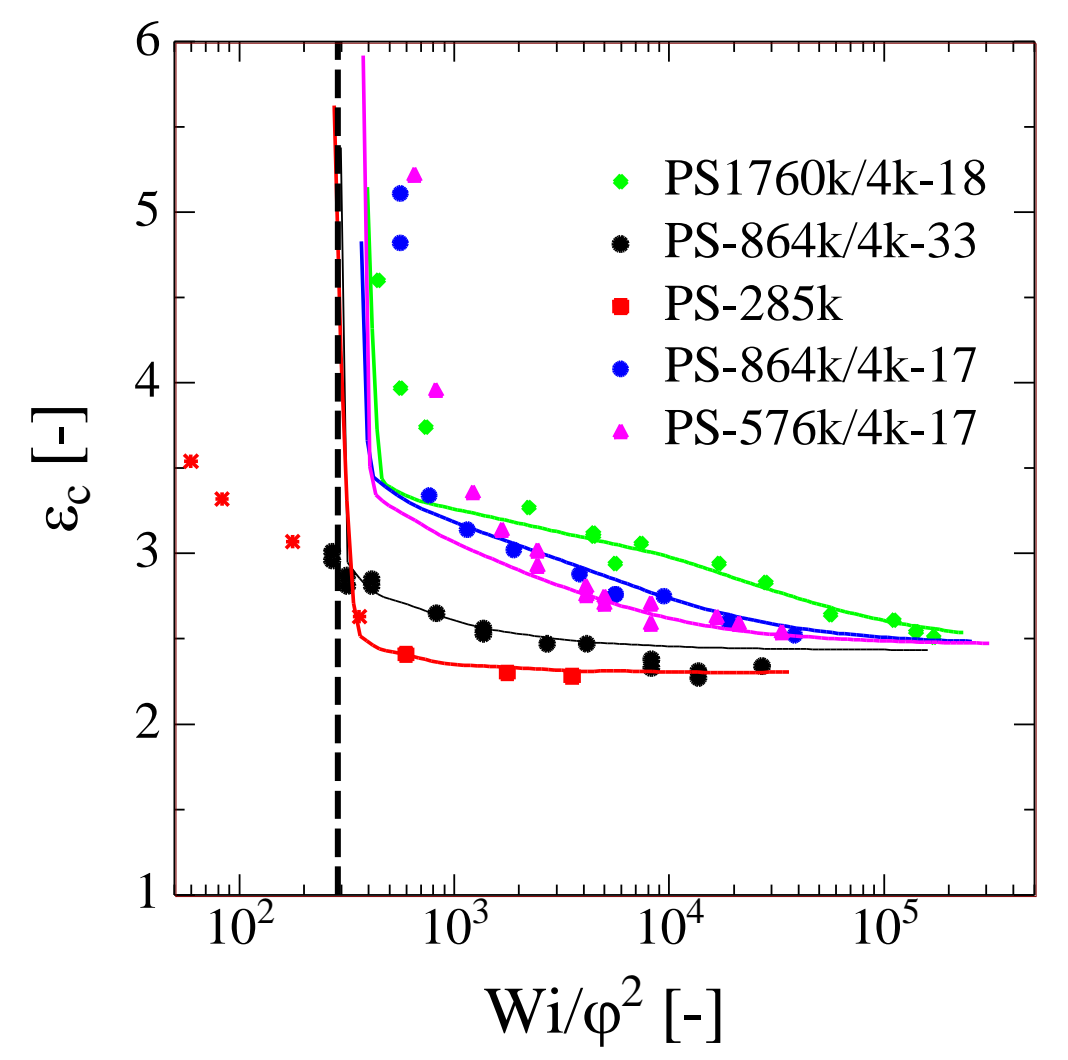

Fig.6: Comparison of data (symbols) and predictions (lines) of critical Hencky strain at break $\varepsilon_{c}$ as a function of reduced Weissenberg number $W i / \varphi^{2}$ for PS melt and solutions. Samples which failed by fast necking are presented as asterisks. Reduced critical Weissenberg number at the onset of fracture $W i_{c} / \varphi^{2}=288$, Eq. (39), is indicated by vertical dotted line.

The scaling of the tensile stress according to relation (38) is presented in Fig. 7 as a function of the Weissenberg number $W i_{e}$ based on the equilibration time $\tau_{e}$ of the entanglement segments. The critical reduced stress at fracture of $\sigma_{c} \varphi / G_{N} \cong 180$ as expected by the scaling relation (38) is indicated by the horizontal dotted line. The experimental data cluster around this line with a trend to increasing $\sigma_{c} \varphi / G_{N}$ with increasing $W i_{e}$. Above $W i_{e}=1$, i.e. above 
the second crossover of G' and G', when approaching the transition to the glassy regime, the cluster of fracture data points narrows and the predictions of $\sigma_{c} \varphi / G_{N}$ converge to a single line.

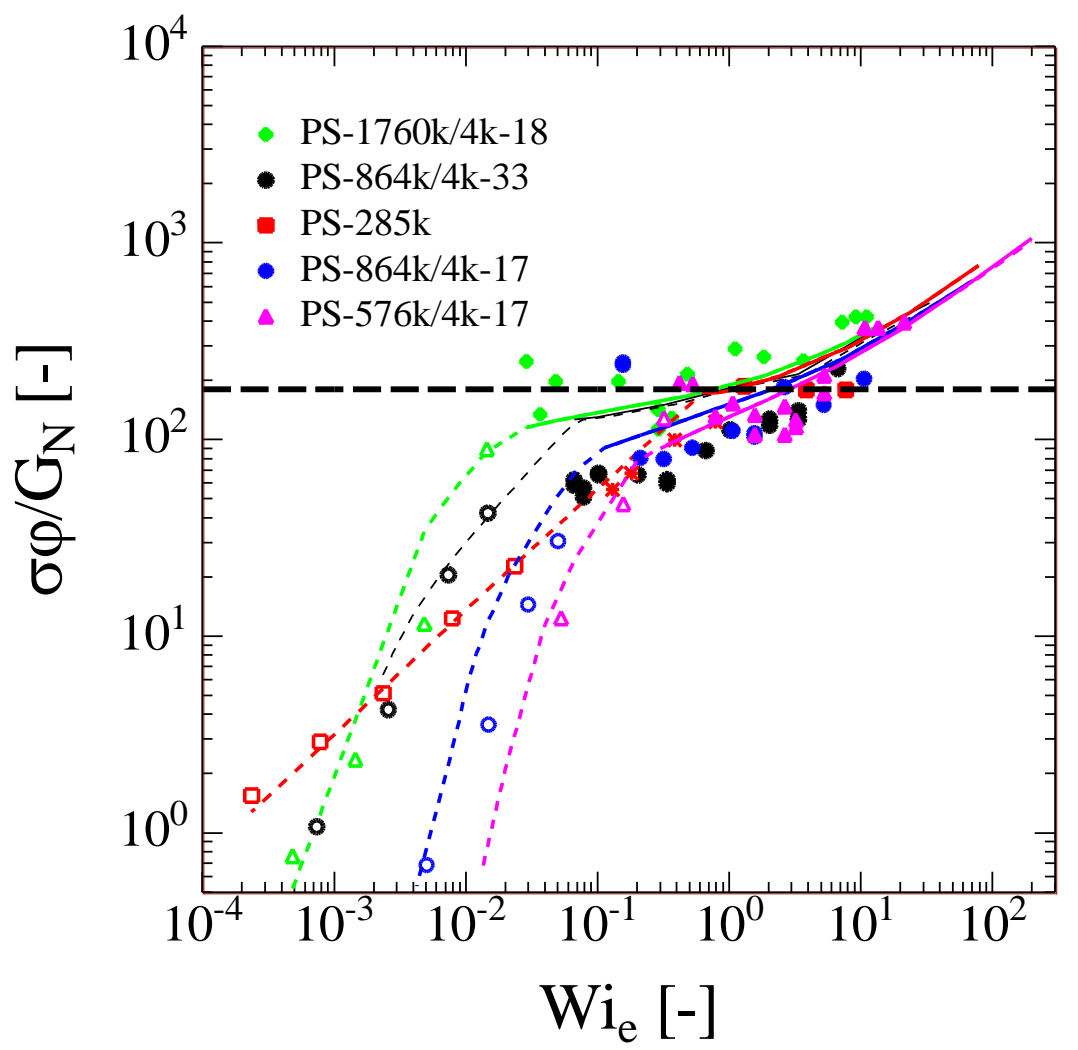

Fig.7: Comparison of data (symbols) and predictions (lines) of reduced tensile stress $\sigma / G_{N}$ as a function of Weissenberg number $W i_{e}=\dot{\varepsilon} \tau_{R} / Z^{2}$ for PS melt and solutions. Fractured samples are indicated by full symbols and full lines, steady-state stress by open symbols and dotted lines. Samples which failed by fast necking are presented as asterisks. The asymptotic critical reduced stress at fracture of $\sigma_{c} \varphi / G_{N} \cong 180$, Eq. (38), is indicated by the horizontal dotted line.

A combination of the scaling of Figs. 6 and 7 is presented in Fig. 8 by plotting the reduced tensile stress $\sigma \varphi / G_{N}$ as a function of the reduced Weissenberg number $\mathrm{Wi} / \varphi^{2}$. No fracture is observed to the left of the vertical dotted line indicating the characteristic number $W i_{c} / \varphi^{2}=288$, while to the right of this line, the critical reduced fracture stress data $\sigma_{c} \varphi / G_{N}$ cluster around the horizontal line with characteristic number $\sigma_{c} \varphi / G_{N}=180$. 


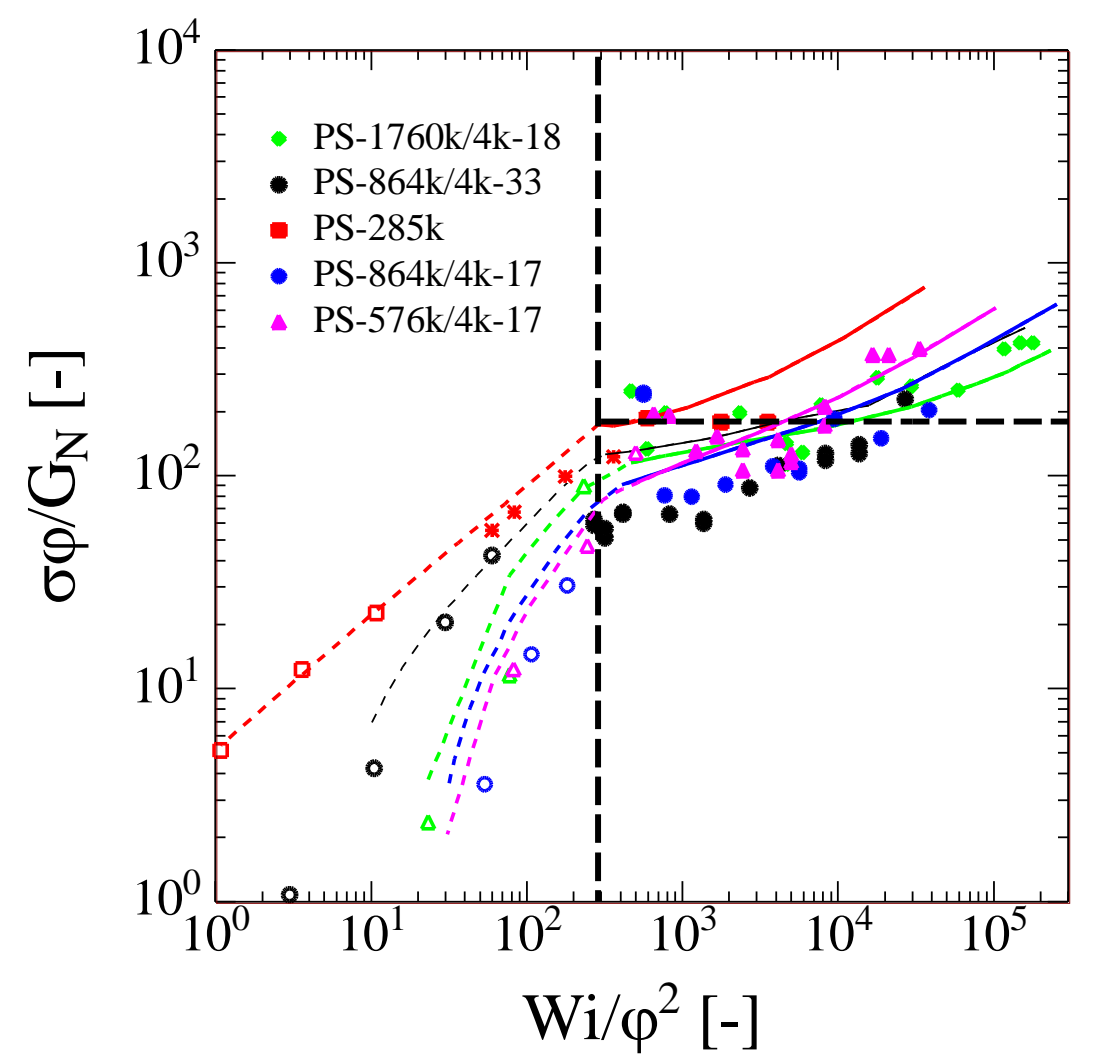

Fig.8: Comparison of data (symbols) and predictions (lines) of reduced tensile stress $\sigma / G_{N}$ as a function of reduced Weissenberg number $W i / \varphi^{2}$ for PS melt and solutions. Fractured samples are indicated by full symbols and full lines, steady-state stress by open symbols and dotted lines. Samples which failed by fast necking are presented as asterisks. The asymptotic critical reduced stress at fracture of $\sigma_{c} \varphi / G_{N} \cong 180$, Eq. (38), is indicated by the horizontal dotted line. Vertical dotted line represents reduced critical Weissenberg number at the onset of fracture $W i_{c} / \varphi^{2}=288$, Eq. (39). 


\section{CONCLUSIONS}

According to our analysis, brittle fracture of entangled polystyrene melts and solutions in homogeneous elongational flow is determined by three characteristic numbers: 36,180 , and 288. These numbers characterise the scaling relations for brittle fracture of the polymer melt and the polymer solutions presented.

The first number relates the thermal energy $3 k T$ to the bond energy $U$ of a carbon-carbon bond with $U / 3 k T \cong 36$. When the strain energy $w\left(N_{e m}\right)=3 k T f \lambda \varphi$ of an entanglement segment with $N_{e m}$ Kuhn monomers reaches the bond energy $U$ of one carbon-carbon bond, the chain fractures and the polymer filament fails by chain scission. In start-up flows, segments with long relaxation times, i.e. those preferably in the middle of the chain, will be the first to reach the critical energy $w_{c}$ and will fracture. This is in agreement with experimental evidence [57-61] as presented in the Introduction. Chain scission is followed by crack initiation and fast crack growth. The product of critical tension $f_{c}$ and critical stretch $\lambda_{c}$ of the entanglement are related to the $\mathrm{C}-\mathrm{C}$ bond energy by

$$
f_{c} \lambda_{c} \varphi \cong \frac{U}{3 k T} \cong 36
$$

Polymer chains at fracture at not fully stretched. This is in contrast to the fracture hypothesis of Lake and Thomas [36] as modified by Mazich and Samus [39] assuming that all $N$ carboncarbon bonds between two entanglement points are fully stretched at fracture, would require $f_{c} \lambda_{c} \varphi=\frac{N U}{3 k T} \cong 36 N$. This is larger by two orders of magnitude than what is observed experimentally for the polystyrene melt and solutions considered.

The second number results from the high Weissenberg number limit of the EIP model: The critical tensile stress at fracture in this limit is $\sigma_{c}=5 G_{N} f_{c} \lambda_{c}$ or

$$
\frac{\sigma_{c} \varphi}{G_{N}} \cong 5 \frac{U}{3 k T} \cong 180
$$


The high Weissenberg number limit of the evolution equation (25) of the EIP model determines the critical Weissenberg number $W i_{c}$ for the onset of fracture, $f_{c} \lambda_{c}=\frac{3}{2} \varphi^{-2} \sqrt{2 W i_{c}}$ , or

$$
\frac{W i_{c}}{\varphi^{2}} \cong \frac{2}{9}\left(\frac{U}{3 k T}\right)^{2} \cong 288
$$

and thereby the third characteristic number. The critical Weissenberg number $W i_{c}$ is proportional to the square of the polymer fraction $\varphi$, i.e. $W i_{c} \cong 288 \varphi^{2}$, and in agreement with experimental evidence, fracture occurs only for elongational flows with $W i_{c} / \varphi^{2} \geq 288$ for all polystyrene systems considered.

The maximum extensibility $\lambda_{\max }=\sqrt{N_{e}}$ of the entanglement segment determines the Hencky strain at fracture $\varepsilon_{c}$ at $W i \geq W i_{c}$. However, at flow rates larger than the inverse equilibration time $\tau_{e}=\tau_{R} / Z^{2}$ of the entanglement segment, the effective finite extensibility of polymer solutions was found to be the same as for melts, i.e. $\lambda_{\max }=\sqrt{N_{e m}}$. This explains why at high $W i$, the Hencky strain at fracture for all samples approaches a value of $\varepsilon_{c} \cong 2.4$. The relevant Weissenberg number is $W i_{e}=\dot{\varepsilon} \tau_{e}=W i / Z^{2}$, and at $W i_{e} \cong 1$, the reduced critical stress at fracture $\sigma_{c} \varphi / G_{N}$ attains a value of approximately 180 for all polystyrene systems considered.

Model predictions and scaling relations are in reasonable agreement with experimental evidence for the polystyrene melt and solutions investigated. With the availability of the filament stretching rheometer with locally controlled elongational strain and strain rate [2], experimental fracture data of other polymers than polystyrene can be expected in the near future, which will allow a critical assessment of the universality of the scaling relations for elongational flow presented. Also, progress in incorporating mechanophoric moieties such as spiropyran in the polymer chain signalling directly molecular forces will even permit a direct determination of the spatial distribution of chain stresses [46-48].

\section{Supplementary Material}

See supplementary material for linear-viscoelastic mastercurves of G' and G', (Fig. S1) as well as parsimonious relaxation spectra (Table S1) for the polystyrene melt and solutions investigated. 


\section{References}

[1] Wagner, M.H., Narimissa, E., and Huang, Q., "On the origin of brittle fracture of entangled polymer solutions and melts," J. Rheology 62, 221-223 (2018).

[2] Huang, Q., Mangnus, M., Alvarez, N. J., Koopmans, R., and Hassager, O., “'A new look at extensional rheology of low-density polyethylene," Rheol. Acta 55, 343-350 (2016).

[3] Huang, Q., Alvarez, N. J., Shabbir, A., and Hassager, O., "Multiple Cracks Propagate Simultaneously in Polymer Liquids in Tension," Physical Review Letters 117, 087801 (2016).

[4] Huang, Q., and Hassager, O., "'Polymer liquids fracture like solids," Soft Matter 13, 3470-3474 (2017).

[5] Malkin, A. Y., and Petrie, C., "Some conditions for rupture of polymer liquids in extension,' J. Rheology 41, 1-25 (1997).

[6] Griffith, A. A., "The phenomena of rupture and flow in solids,' Philosophical transactions of the royal society of London. Series A 221, 163-198 (1921).

[7] Wang, Y., and Wang, S.-Q., "Salient features in uniaxial extension of polymer melts and solutions: Progressive loss of entanglements, yielding, non-Gaussian stretching, and rupture," Macromolecules 44, 5427-5435 (2011).

[8] Huang, Q., "Exploring the mechanism of fracture for entangled polymer liquids in extensional flow," Physics of Fluids 31, 083105 (2019).

[9] Baumgaertel, M.; Schausberger, A.; Winter, H. H., "The relaxation of polymers with linear flexible chains of uniform length," Rheol. Acta 29, 400-408 (1990).

[10] Huang, Q.; Mednova, O.; Rasmussen, H. K.; Alvarez, N. J.; Skov, A. L.; Almdal, K.; Hassager, O., "Concentrated polymer solutions are different from melts: Role of entanglement molecular weight," Macromolecules 46, 5026-5035 (2013).

[11] Huang, Q.; Alvarez, N. J.; Matsumiya, Y.; Rasmussen, H. K.; Watanabe, H.; Hassager, O., "Extensional rheology of entangled polystyrene solutions suggests importance of nematic interactions," ACS Macro Letters 2, 741-744 (2013).

[12] Winter, H. H.; Mours, M., "The cyber infrastructure initiative for rheology;" Rheol. Acta 45, 331-338 (2006).

[13] Dealy, J. M.; Read, D. J.; Larson, R. G., Structure and rheology of molten polymers: from structure to flow behavior and back again. Carl Hanser Verlag GmbH Co KG: 2018.

[14] Osaki, K.; Nishizawa, K.; Kurata, M., "Material time constant characterizing the nonlinear viscoelasticity of entangled polymeric systems," Macromolecules 15, 1068-1071 (1982). 
[15] Takahashi, M.; Isaki, T.; Takigawa, T.; Masuda, T., "Measurement of biaxial and uniaxial extensional flow behavior of polymer melts at constant strain rates," J. Rheology 37, 827-846 (1993).

[16] Isaki, T.; Takahashi, M.; Urakawa, O., "Biaxial damping function of entangled monodisperse polystyrene melts: Comparison with the Mead-Larson-Doi model," J. Rheology 47, 1201-1210 (2003).

[17] Menezes, E.; Graessley, W., "Nonlinear rheological behavior of polymer systems for several shear-flow histories,” J. Poly. Sci. Part B: Poly. Phys. 20, 1817-1833 (1982).

[18] Wagner, M. H., "Scaling relations for elongational flow of polystyrene melts and concentrated solutions of polystyrene in oligomeric styrene," Rheol. Acta 53, 765-777 (2014).

[19] Narimissa, E.; Huang, Q.; Wagner, M. H., "Elongational rheology of polystyrene melts and solutions: Concentration dependence of the interchain tube pressure effect," J. Rheology 64, 95-110 (2020).

[20] Narimissa, E.; Schweizer, T.; Wagner, M. H., "A constitutive analysis of nonlinear shear flow", Rheol. Acta 59, 487-506 (2020).

[21] Wagner, M.H., "The nonlinear strain measure of polyisobutylene melt in general biaxial flow and its comparison to the Doi-Edwards model," Rheol. Acta 29, 594-603 (1990).

[22] Wagner, M.H. and J. Schaeffer, "Nonlinear measures for general biaxial extension of polymer melts," J. Rheol. 36, 1-26 (1992).

[23] Wagner, M.H., P. Rubio and H. Bastian, "The molecular stress function model for polydisperse polymer melts with dissipative convective constraint release," J. Rheol. 45, 1387-1412 (2001).

[24] Wagner, M.H., M. Yamaguchi and M. Takahashi, "Quantitative assessment of strain hardening of low-density polyethylene melts by the molecular stress function model," J. Rheol. 47, 779-793 (2003).

[25] Wagner, M.H., S. Kheirandish and O. Hassager, "Quantitative Prediction of Transient and Steady-State Elongational Viscosity of Nearly Monodisperse Polystyrene Melts," J. Rheol. 49, 1317-1327 (2005).

[26] Rolon-Garrido, V.H., Wagner, M.H., Luap, C., and Schweizer, T., "Modeling nonGaussian extensibility effects in elongation of nearly monodisperse polystyrene melts," J. Rheology 50, 327-340 (2006).

[27] Doi, M. and S.F. Edwards, "Dynamics of Concentrated Polymer Systems. Part 3.- The Constitutive Equation,” J. Chem. Soc., Faraday Trans. 74, 1818-1832 (1978).

[28] Doi, M. and S.F. Edwards, "Dynamics of Concentrated Polymer Systems. Part 4.Rheological Properties,” J. Chem. Soc., Faraday Trans. 75, 38-54 (1979).

[29] Bird, R.B., Ch.F. Curtiss, R.C. Armstrong and O. Hassager, Dynamics of Polymeric Liquids Vol. 2. Kinetic Theory, Wiley and Sons, USA, 1987. 
[30] Larson, R.G., Constitutive Equations for Polymer Melts, Butterworths, Stoneham, 1988.

[31] Cohen, A., "A Padé approximant to the inverse Langevin function," Rheol. Acta 30, 270273 (1991).

[32] Ye, X. and T. Sridhar, "Effects of the Polydispersity on Rheological Properties of Entangled Polystyrene Solutions,” Macromolecules 38, 3442-3449 (2005).

[33] Marrucci, G., and Hermans, J. J., "Nonlinear viscoelasticity of concentrated polymeric liquids," Macromolecules 13, 380-387 (1980).

[34] De Gennes, P., "Coil-stretch transition of dilute flexible polymers under ultrahigh velocity gradients,'” J. Chem. Phys. 60, 5030-5042 (1974).

[35] Marrucci, G., "Molecular modelling of flows of concentrated polymers." Transport Phenomena in Polymeric Systems (Wiley, 1989).

[36] Lake G.J., and A.G. Thomas, "The strength of highly elastic materials", Proc. R. Soc. Lond. A 300, 108-119, (1967)

[37] Bhowmick, A., Gent, A., and Pulford, C., "Tear strength of elastomers under threshold conditions,' Rubber Chem. \& Tech. 56, 226-232 (1983).

[38] Grandbois, M., Beyer, M., Rief, M., Clausen-Schaumann, H., and Gaub, H. E., "How strong is a covalent bond?,' Science 283, 1727-1730 (1999).

[39] Mazich, K. A., and Samus, M., "'Role of entanglement couplings in threshold fracture of a rubber network," Macromolecules 23, 2478-2483 (1990).

[40] Wagner, M. H., "Scaling relations for elongational flow of polystyrene melts and concentrated solutions of polystyrene in oligomeric styrene," Rheol. Acta 53, 765-777 (2014).

[41] Howard, R.M., "Analytical approximations for the inverse Langevin function via linearization, error approximation, and iteration," Rheol. Acta 59, 521-544 (2020).

[42] Wagner, M.H., Narimissa, E., and Huang, Q., "Response to "Letter to the Editor: 'Melt rupture unleashed by few chain scission events in fully stretched strands" " [J. Rheol. 63, 105 (2018)], " J. Rheology 63, 419-421 (2019).

[43] Ligoure, C., and Mora, S., "Fractures in complex fluids: the case of transient networks," Rheol. Acta 52, 91-114 (2013).

[44] Wang, S., Panyukov, S., Rubinstein, M., Craig, S.L., "Quantitative Adjustment to the Molecular Energy Parameter in the Lake-Thomas Theory of Polymer Fracture Energy," Macromolecules 52, 2772-2777 (2019).

[45] Vernerey, F.J., Brighenti, R., Long, R., Shen, T., "Statistical Damage Mechanics of Polymer Networks," Macromolecules 51, 6609-6622 (2018). 
[46] Chen, Y., Spiering, A.J.H., KarthikeyanS, Peters, G.W.M., Meijer, E.W., Sijbesma, R.P., "Mechanically induced chemiluminescence from polymers incorporating a 1,2-dioxetane unit in the main chain," Nature Chemistry 4, 559-562 (2012).

[47] Jiang, S., Zhang, L., Xie, T., Lin, Y., Zhang, H., Xu, Y., Weng, W., Dai, L., "Mechanoresponsive PS-PnBA-PS Triblock Copolymers via Covalently Embedding Mechanophore," ACS Macro Letters 2, 705-709 (2013).

[48] Chen, Y., Yeh, C.J., Qi, Y., Long, R., Creton, C., "From Force Responsive Molecules to Quantifying and Mapping Stresses in Soft Materials," Science Advances 6, eaaz5093 (2020).

[49] Sakaguchi, M., and Sohma, J. "ESR Evidence for Main-Chain Scission Produced by Mechanical Fracture of Polymers at Low Temperature," J. Polymer Sci., Polymer Physics Edition 13, 1233-1245 (1975).

[50] Fordyce, P., Defries, K.L., and Franconi, B.M. "Chain scission and mechanical degradation of polystyrene," PES 6, 421-427 (1984).

[51] Mikos, A.G. and Peppas, N.A "Polymer chain entanglements and brittle fracture," J. Chern. Phys. 88, 1337-1342 (1988).

[52] Zhurkov, S.N. and Korsukov V.E. "Atomic Mechanism of Fracture of Solid Polymers," J. Polym. Sci., Polymer Physics Edition 12, 385-398 (1974).

[53] Tabata, M., Hosokawa, Y., Watanabe, O., and Sohma, J., "Direct Evidence for Main Chain Scissions of Polymers in Solution Caused by High Speed Stirring," Polymer Journal, 18, 699-712 (1986).

[54] Rehner, J. "Birefringence of Polyisobutylene in Solution and the Frenkel Theory of Macromolecular Rupture,” J. Chem. Phys. 13, 450 (1945).

[55] Bestul, A.B. "Kinetics of Capillary Shear Degradation in Concentrated Polymer Solutions," J. Chem. Phys. 24, 1196-1201 (1956).

[56] Bestul, A.B. "Energy Requirements of Mechanical Shear Degradation in Concentrated Polymer Solutions," J. Chem. Phys. 32, 350-356 (1960).

[57] Ballauf, M. and Wolf, B.A. "Degradation of Chain Molecules. 2. Thermodynamically Induced Shear Degradation of Dissolved Polystyrene,"Macromolecules 17, 209-216 (1984).

[58] González-González, V.A., Neira-Velázquez, G. and Angulo-Sánchez, J.L. "Polypropylene chain scissions and molecular weight changes in multiple extrusion," Polymer Degradation and Stability 60, 33-42 (1998).

[59] Canevarolo, S.V., "Chain scission distribution function for polypropylene degradation during multiple extrusions," Polymer Degradation and Stability 70, 71-76 (2000).

[60] Cáceres, C.A. and Canevarolo, S.V. "Calculating the chain scission distribution function (CSDF) using the concentration method," Polymer Degradation and Stability 86, 437-444 (2004). 
[61] da Costa, H.M., Ramos, V.D., and Rocha, M.C.G. "Rheological properties of polypropylene during multiple extrusion," Polymer Testing 24 86-93 (2005).

[62] Bueche, F. "Mechanical degradation of high polymer," J. Appl. Polym:Sci. 4, 101-105 (1960). 

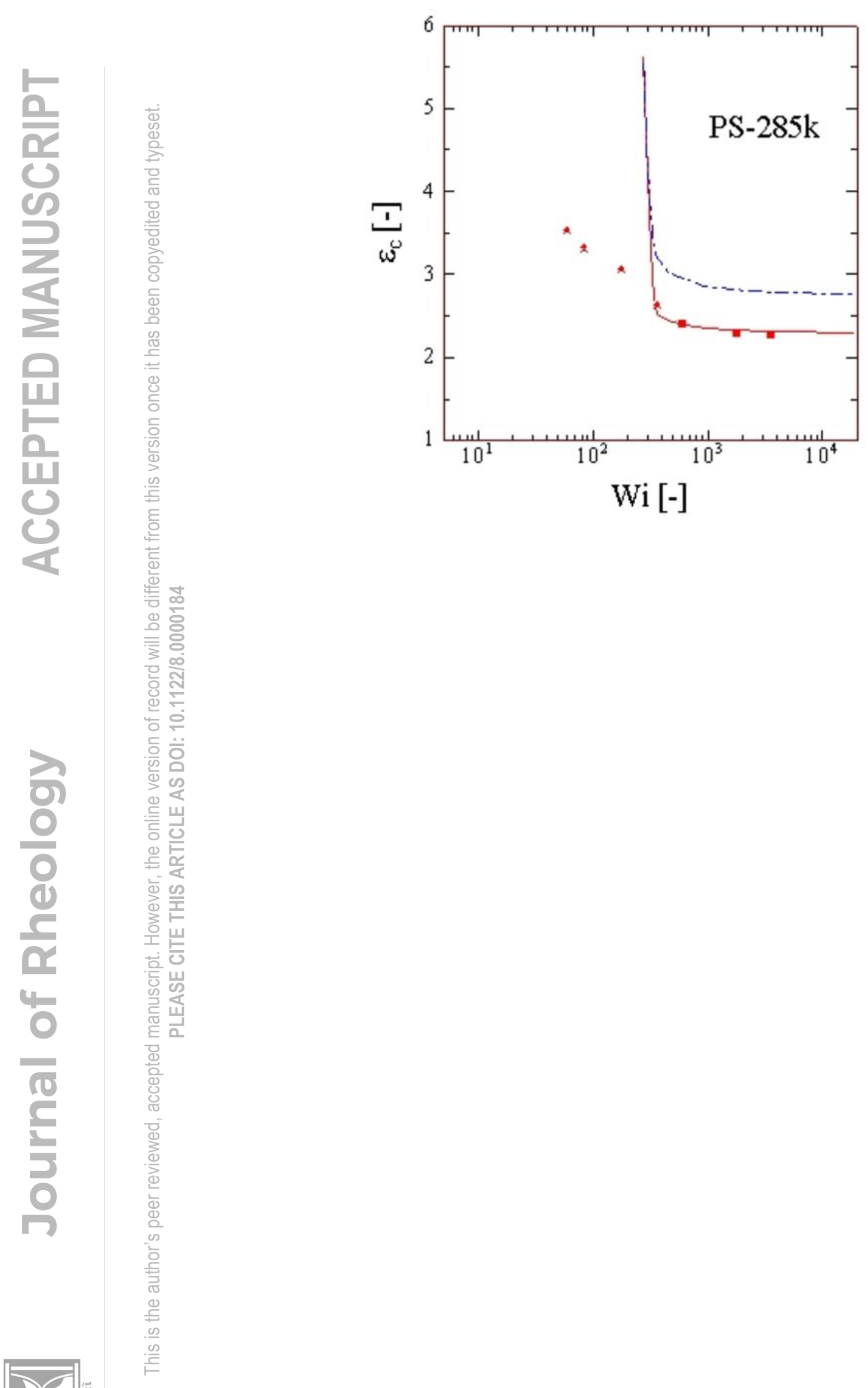


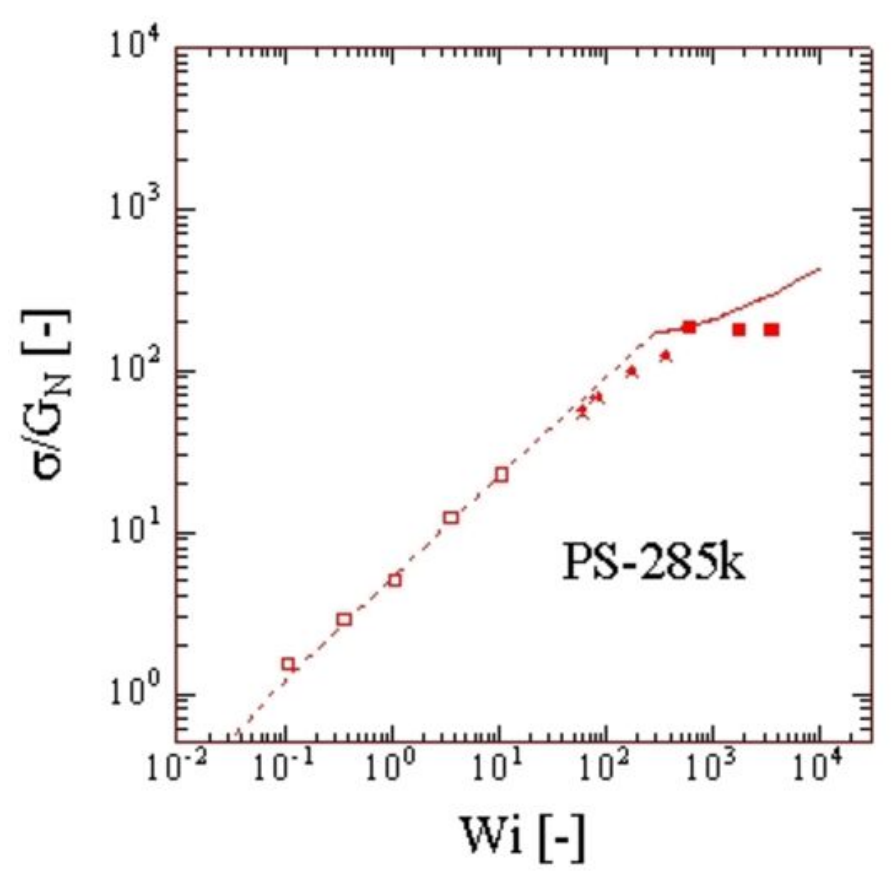




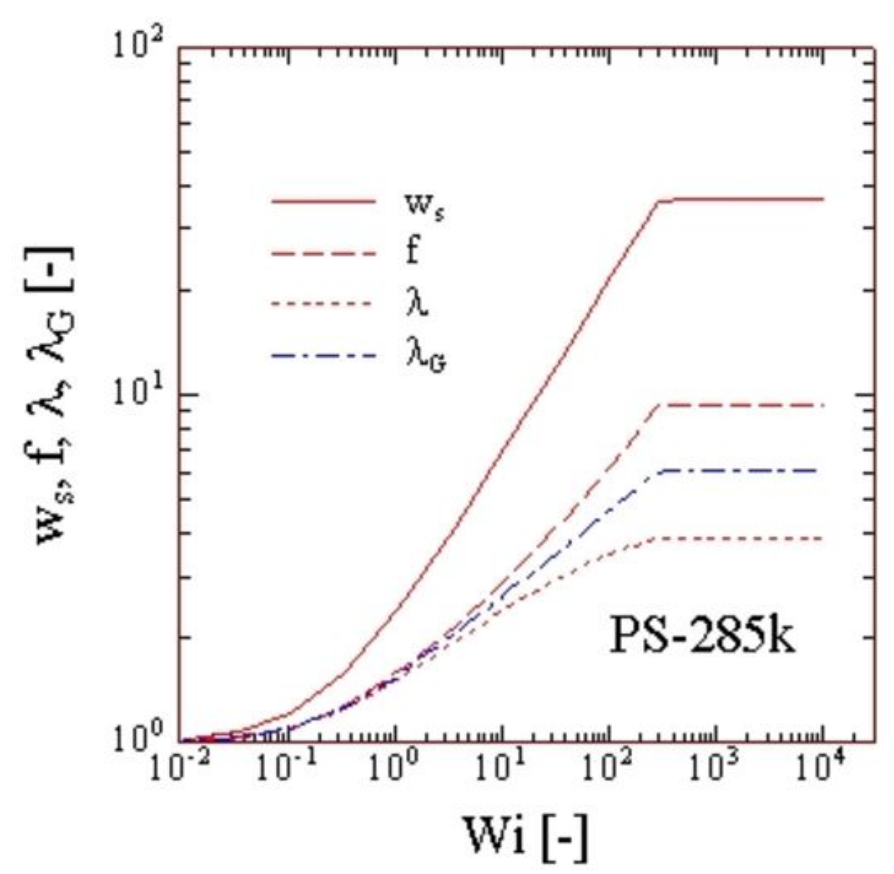




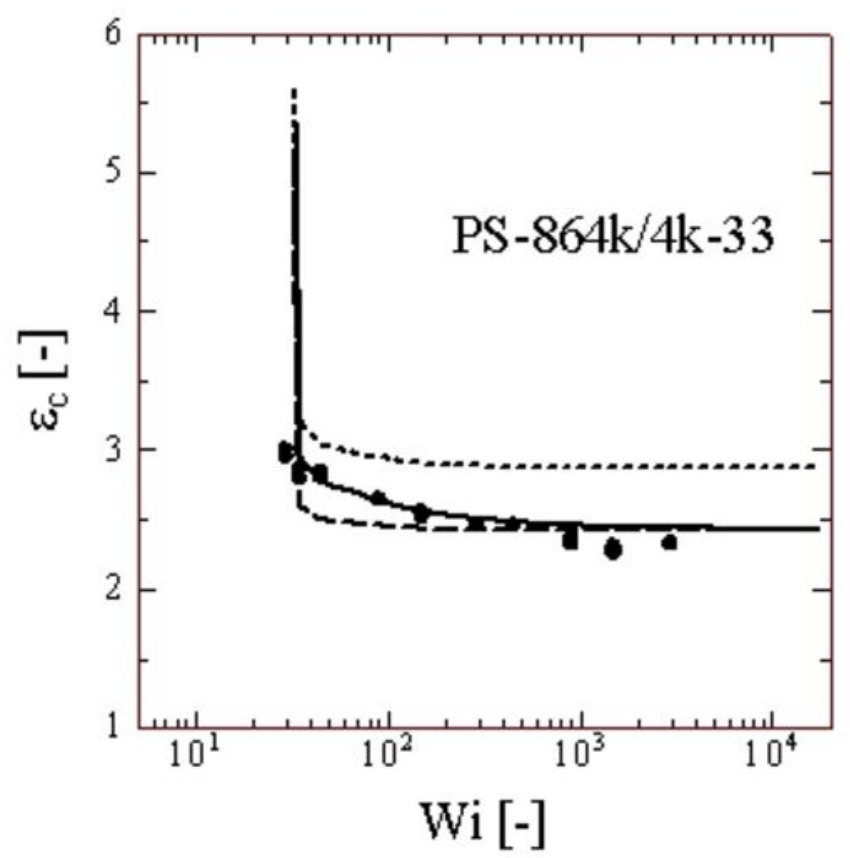




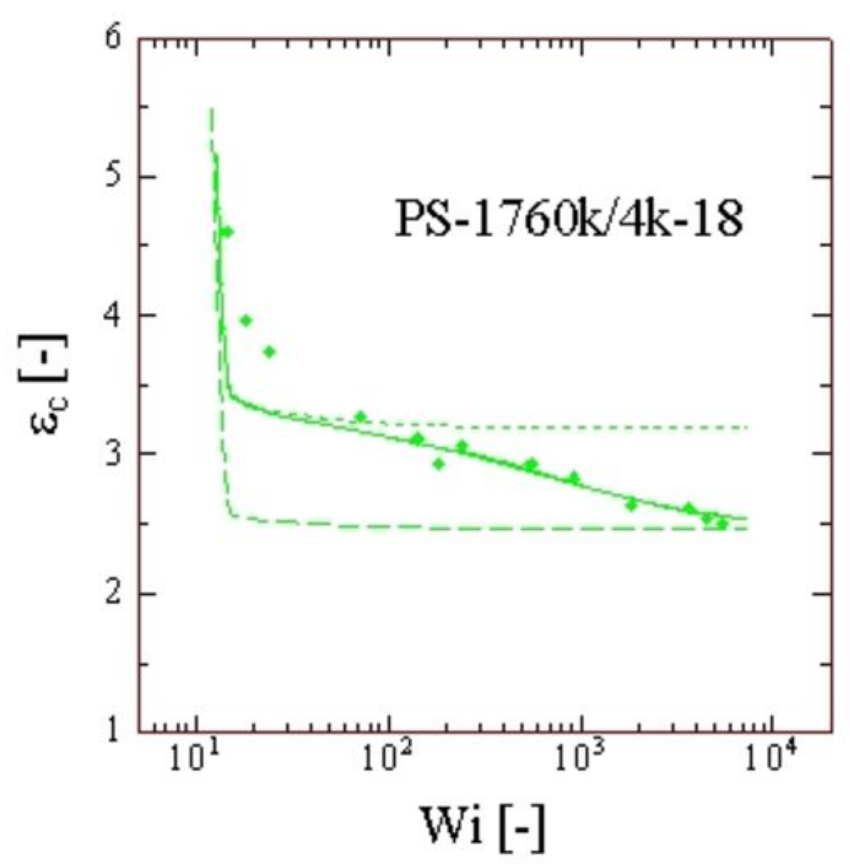




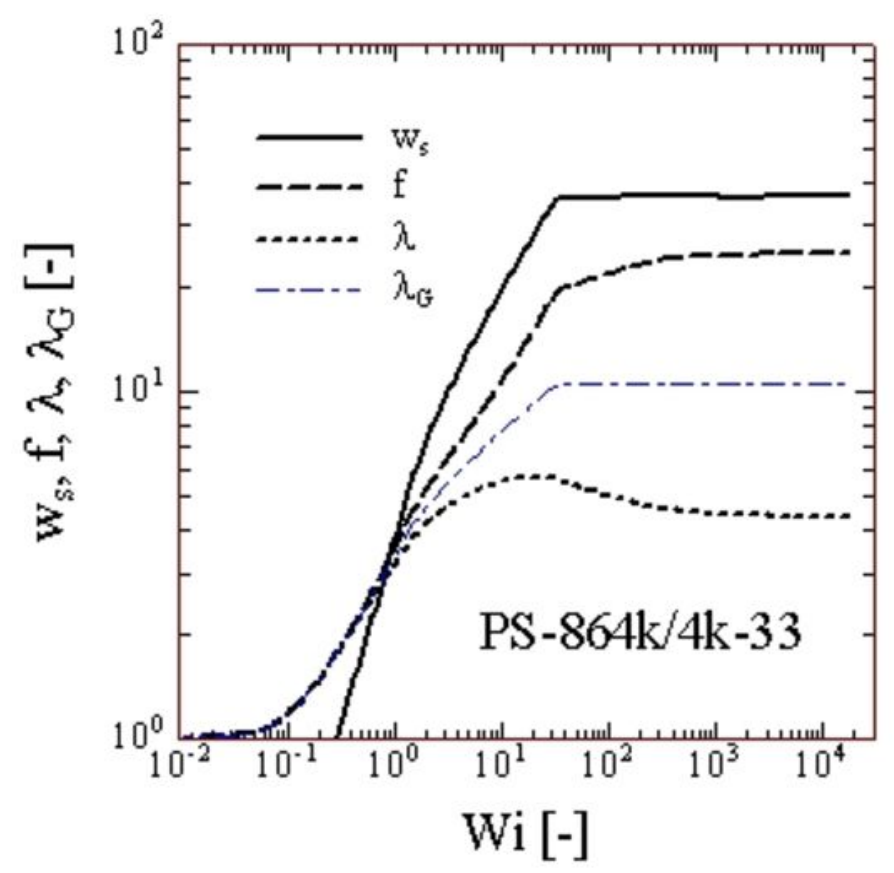




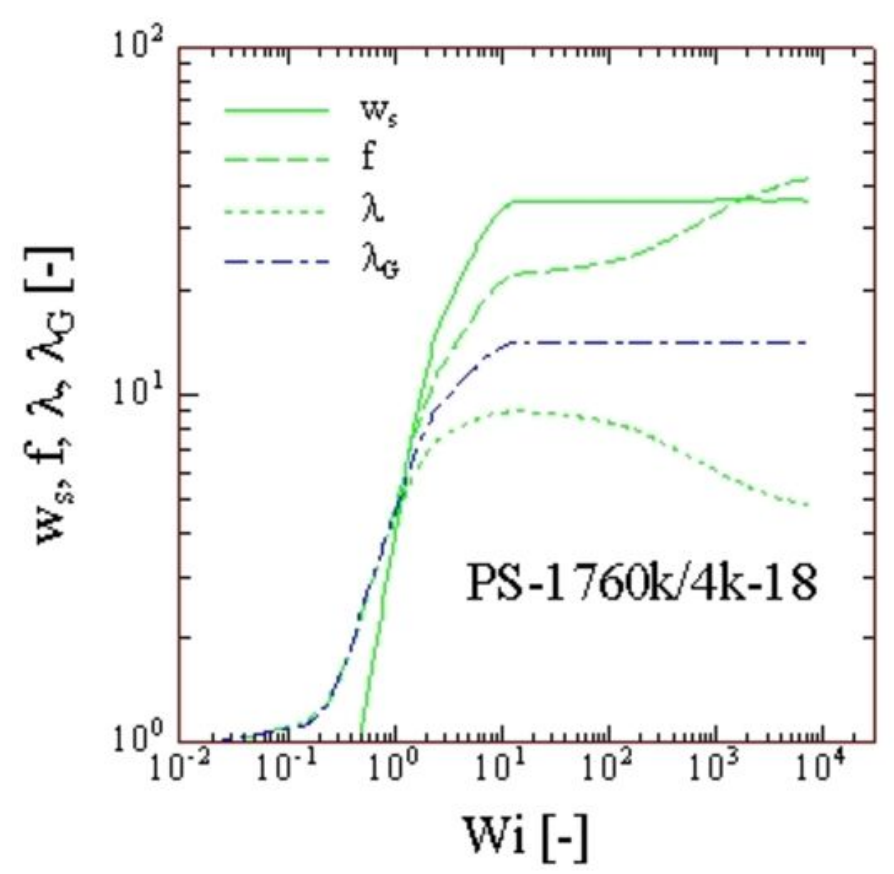




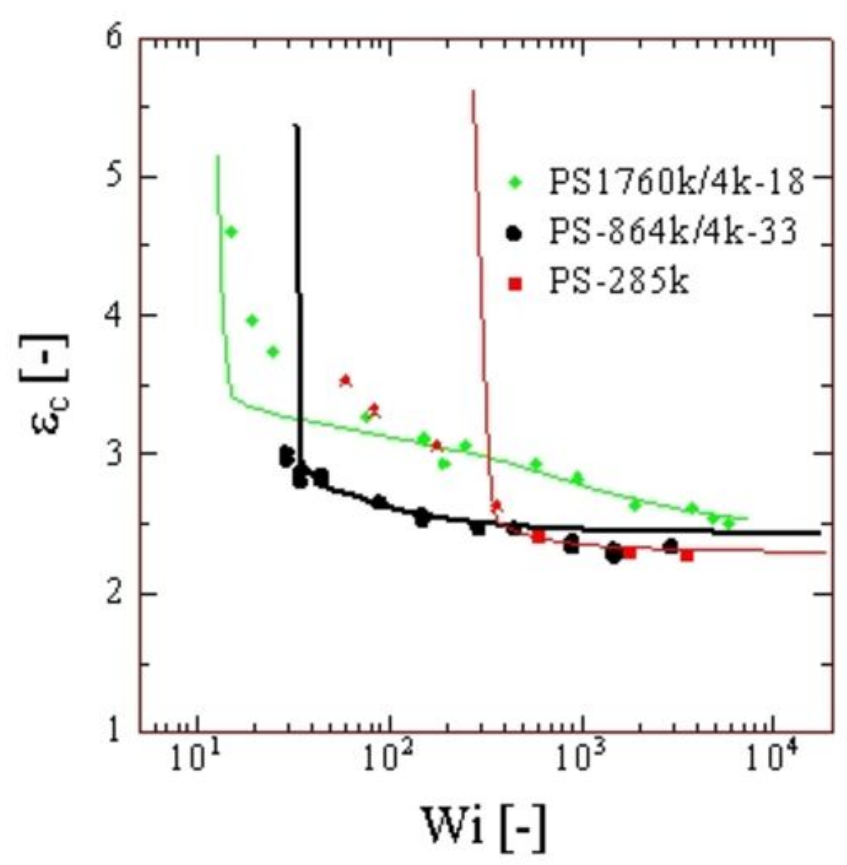




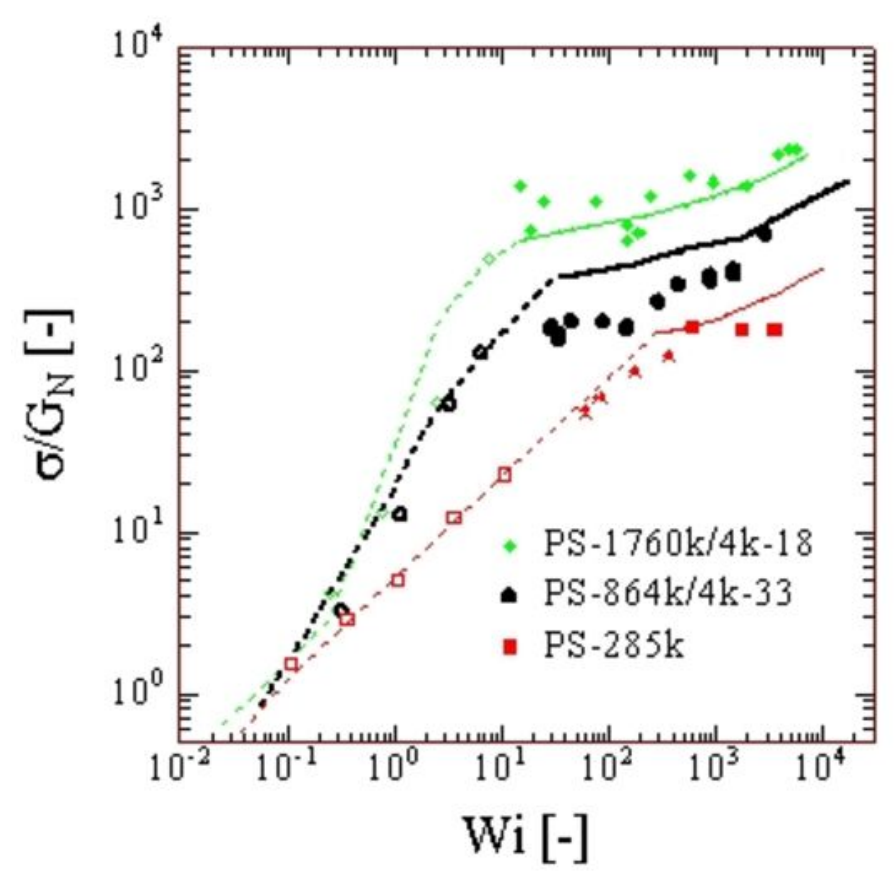




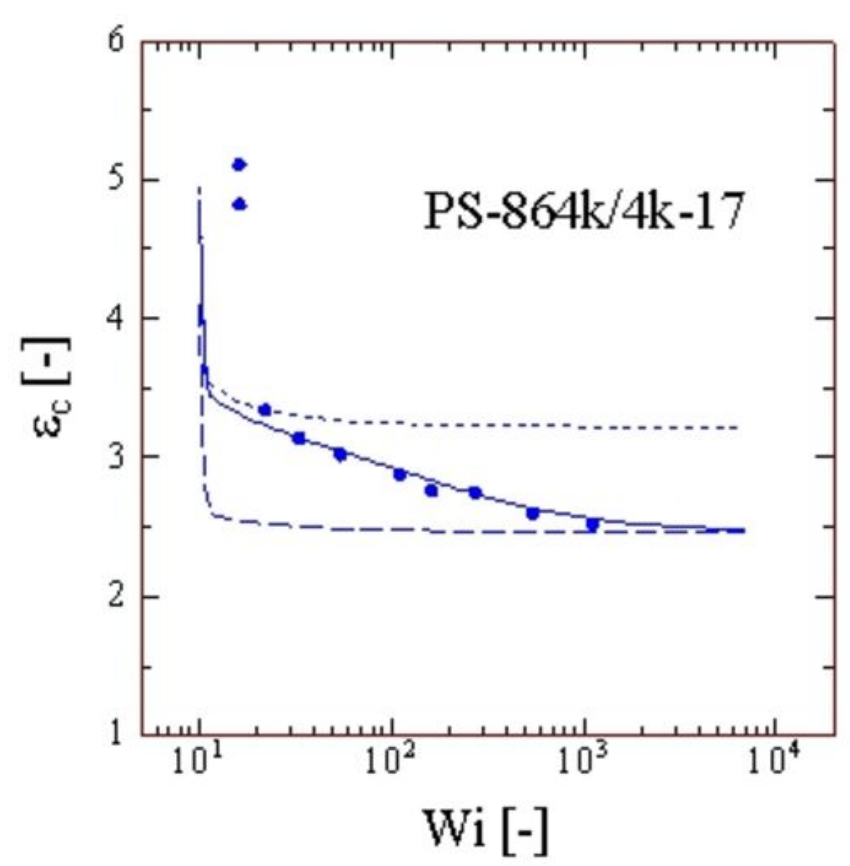




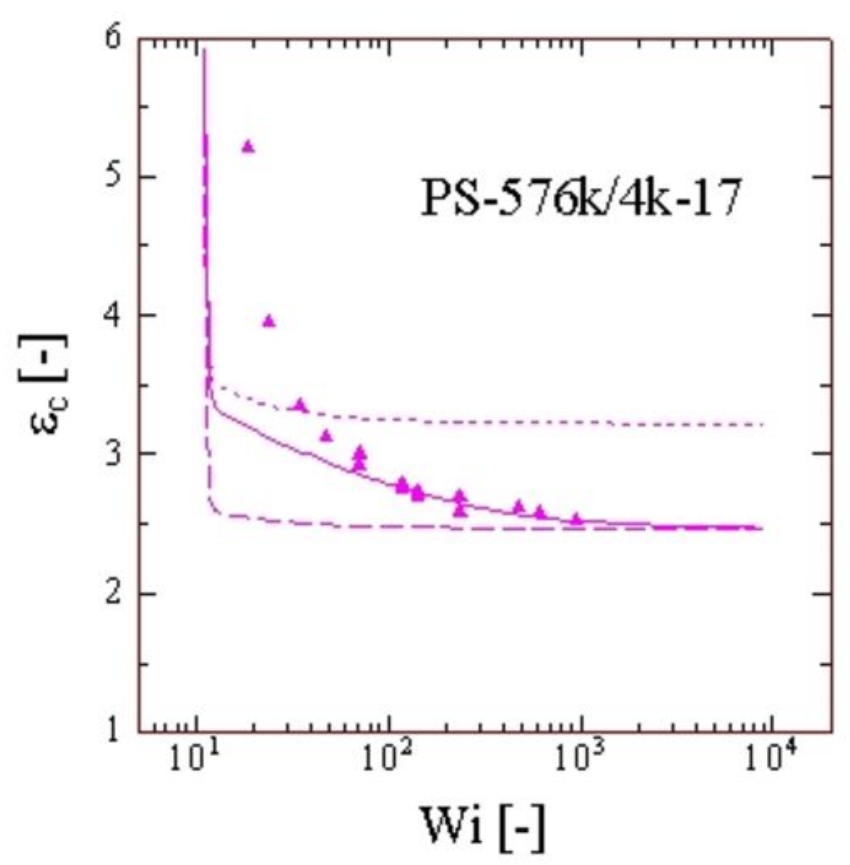




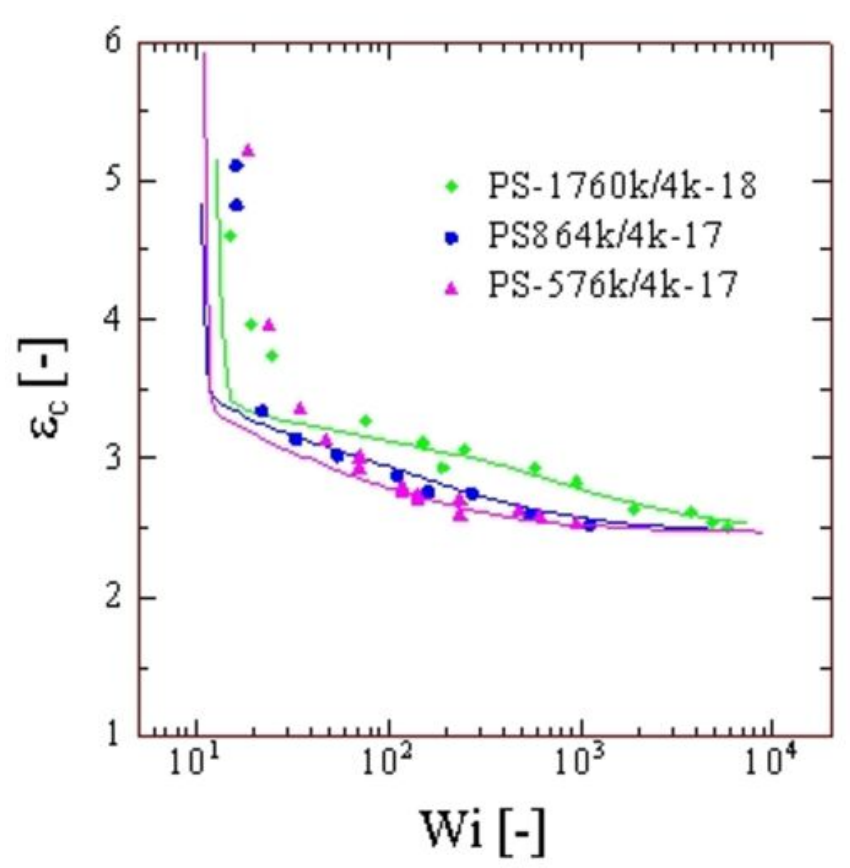




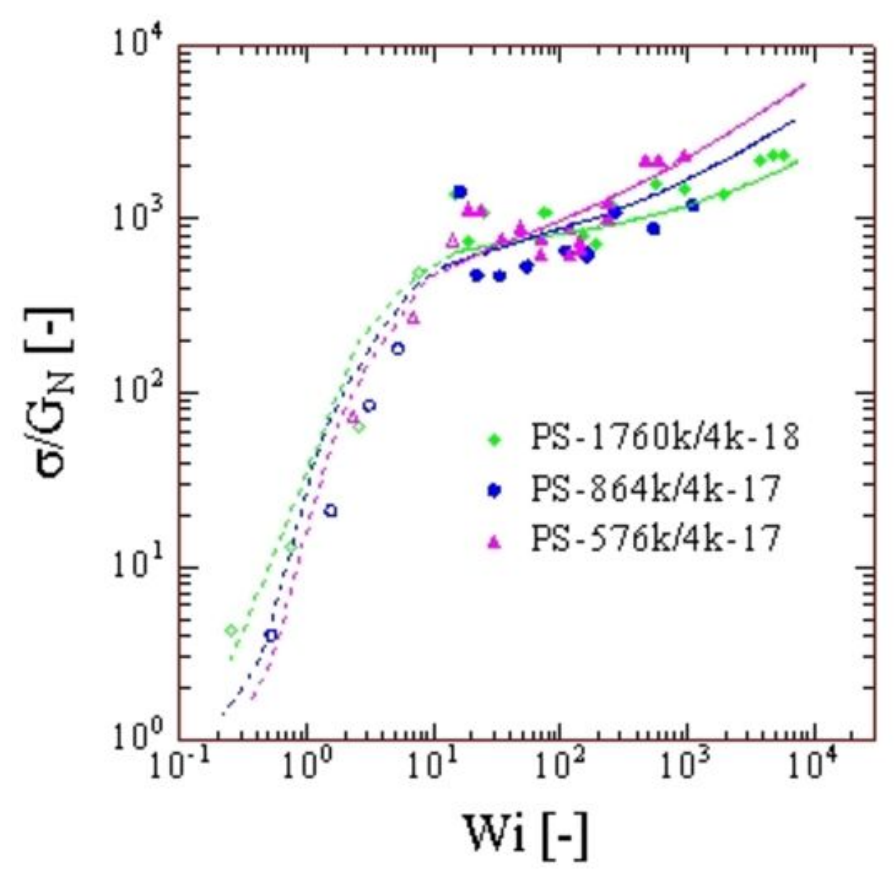




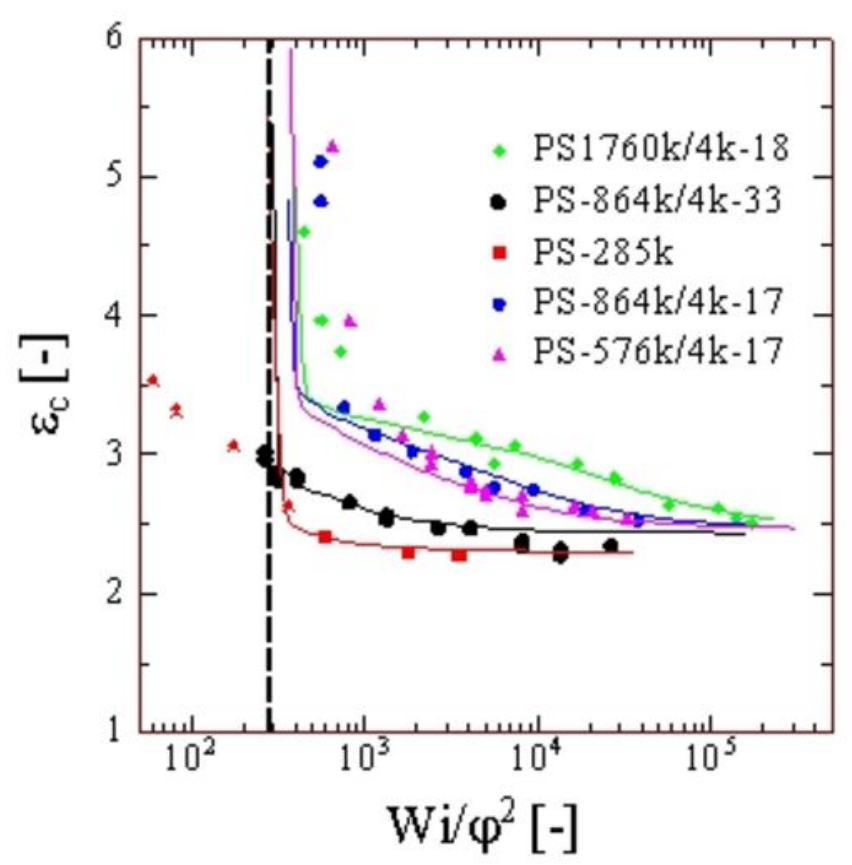




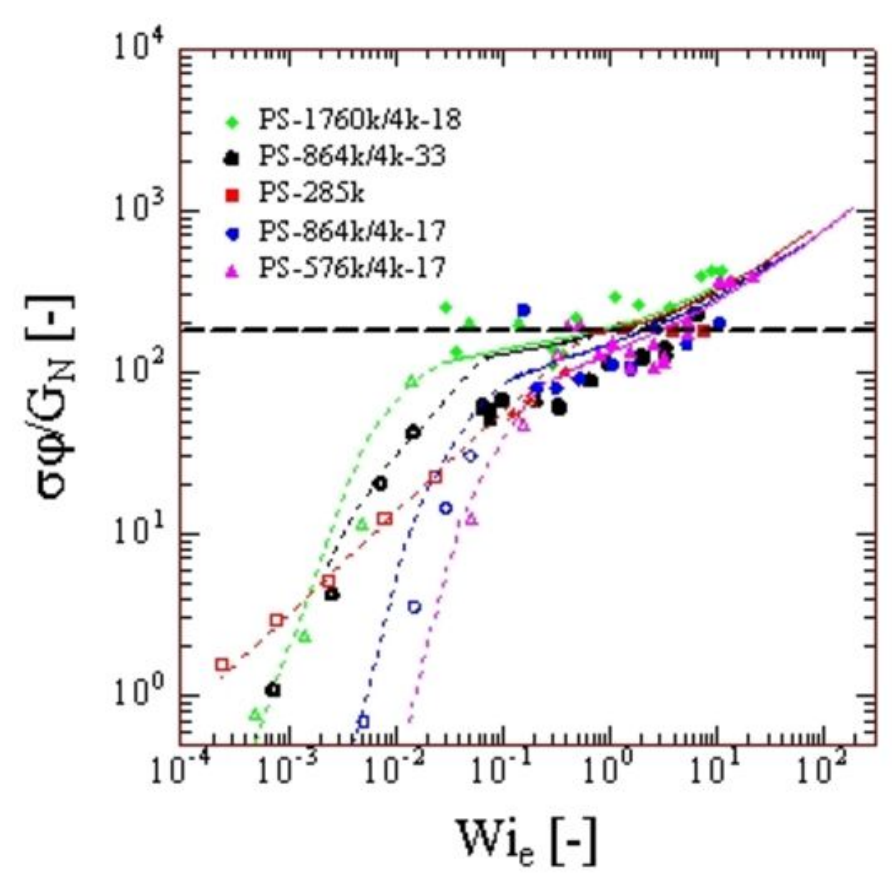




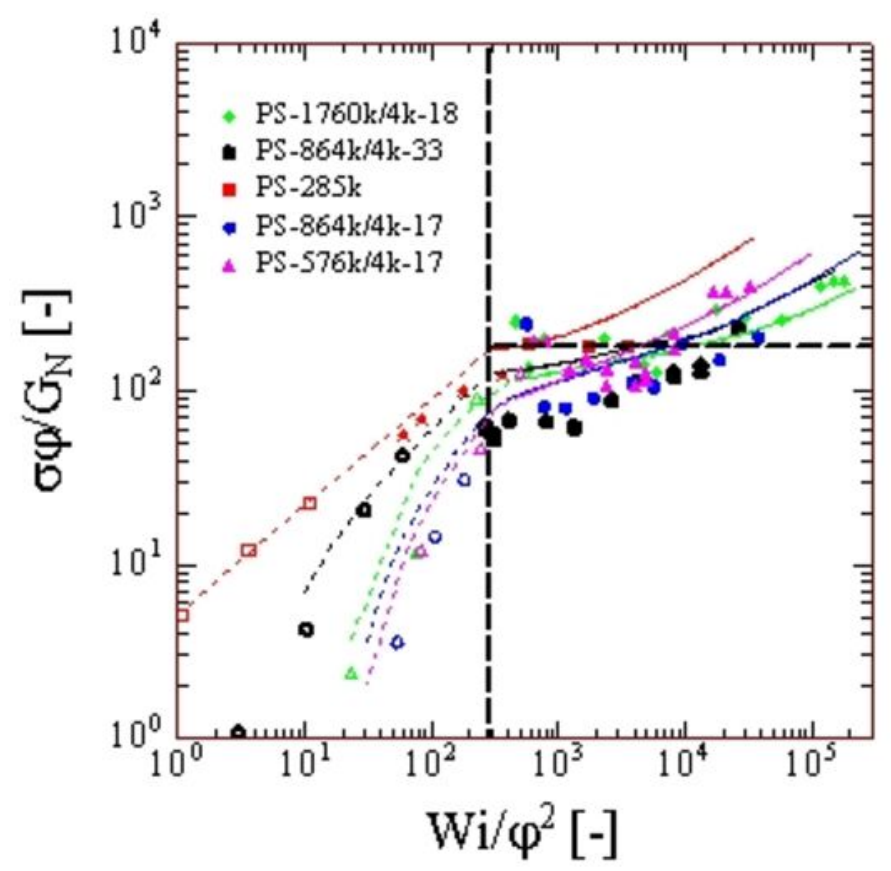

